\title{
Gemeinde überzeugen. Das persuasive Moment des evangelischen Gottesdienstes
}

\section{Einleitung}

An jedem Sonn- und Feiertag werden in Deutschland durchschnittlich 18.000 evangelische Gottesdienste mit insgesamt rund 850.000 Besucherinnen und Besuchern gefeiert (Evangelische Kirche in Deutschland 2014:13f.). Unbestritten geht es in diesen Veranstaltungen um Feier und Andacht, um das Erleben von Gemeinschaftsgefühl und die Unterbrechung des Alltags. Aber geht es in einem evangelischen Gottesdienst darum, die Hörerschaft zu überzeugen, wie es der Titel dieses Beitrags unterstellt? Dies zu behaupten, ist entweder banal oder gewagt. Banal ist es, wird der Gottesdienst - insbesondere der evangelische Gottesdienst in seiner hegemonialen landeskirchlichen Form am Sonntagvormittag - allein mit der Predigt identifiziert. Die Predigt ist eine religiöse Rede und zielt als solche auf Zustimmung zur Auslegung eines Textes, zur Auslegung der Hörerrealität und zur damit verbundenen Gegenwartsanalyse (pointiert formuliert das etwa Gräb 2013:39-42). Damit ist sie dem Eigenanspruch nach mehr als Informationsvermittlung. Sie zielt auf die Erschließung von Gewissheit in Bezug auf Glaubensüberzeugungen und will also auf Glaubensgewissheit hinwirken. Gewagt ist die einleitende Behauptung, wenn der Gottesdienst mit der Liturgie gleichgesetzt und also unter Ausschluss der Predigt betrachtet wird. Es bleibt eine Vielzahl historisch gewachsener Stücke, mehr oder weniger formelhafte Sprache, Gesang und Stille. Der mit der Liturgie identifizierte Gottesdienst wird dabei, mehr oder weniger metaphorisch gesprochen, als Gespräch zwischen Gott und Mensch unter Ausschluss der Kommunikation zwischen Menschen definiert. Als Vorgang, der formal ausschließlich Altbekanntes wiederholt, kann er kaum persuasiv sein. 
Die Frage, ob es sich bei einem Gottesdienst als Ganzem, der Liturgie und Predigt integriert, um ein zumindest phasenweise persuasives Geschehen handelt, wie sich ferner dabei das Persuasive zum Anderen seiner selbst verhält, ist zu ihrer Beantwortung auf empirische Daten sowie auf ihre hermeneutische Fundierung und Interpretation angewiesen. Und nicht zuletzt auf einen Persuasionsbegriff, der dem Gegenstand gerecht wird. Die folgenden Ausführungen sind ein erster Schritt auf diesem Weg. In einem ersten Durchgang sollen die Bedingungen geklärt werden, die die Rede vom Gottesdienst als Ort der Persuasion ermöglichen. Dazu werden theologische, rhetoriktheoretische und ritualtheoretische Perspektiven aufeinander bezogen. Ein zweiter Durchgang nähert sich dann an empirisch gewonnene Daten, an denen gezeigt wird, wie in der Mikro- und Makroperspektive Persuasion in evangelischen Gottesdiensten zu beobachten ist.

\section{Zur Möglichkeit des persuasiven Moments des evangelischen Gottesdienstes}

Um persuasives Handeln im evangelischen Gottesdienst betrachten zu können, soll zunächst in einigen Schlaglichtern die Beziehung der theologischen und sprechwissenschaftlich-rhetorischen Forschung zur Rhetorizität des Gottesdienstes dargestellt werden. Danach soll ein Zugang zum Persuasionsbegriff erschlossen werden. Schließlich soll anhand der Ritualtheorie von Charlotte Humphrey und James Laidlaw (Humphrey/Laidlaw 1994) die Möglichkeit eröffnet werden, angemessen von Persuasion als Moment des evangelischen Gottesdienstes zu sprechen. In diesem dritten Zugang wird eine Perspektive auf die Unterschiedlichkeit liturgischer Formen entwickelt.

\subsection{Erster Zugang: Kurzer historischer und systematischer Abriss zur Deutung des evangelischen Gottesdienstes}

Am Anfang des Nachdenkens darüber, was (evangelischer) Gottesdienst ist, steht Martin Luther. Er konstatiert im Jahr 1544 zur Einweihung der Schlosskirche zu Torgau, dass ,nichts anderes darin geschehen möge, als dass unser lieber Herr selbst mit uns rede durch sein heiliges Wort und wir wiederum mit ihm reden durch Gebet und Lobgesang“ (Luther 1912:588, vom Verfasser aus dem Frühneuhochdeutschen übertragen). Über Jahrhunderte bis in die Gegenwart wird ein solcher normativer Zugriff auf das Geschehen Gottesdienst perpetuiert (vgl. etwa Graff 1994:1), meist unter Rückgriff auf eben dieses Zitat. Die Norm, die hier etabliert wird, hat ihre Funktion. Sie soll Orientierungswissen für die am evangelischen Gottesdienst Beteiligten bereitstellen. Dieses Orientierungswissen ist vor 
allem für Pfarrerinnen und Pfarrer wichtig und schlägt sich in Agendenwerken, also mehr oder weniger normativen Ablaufplänen für Gottesdienste nieder, etwa dem Gottesdienstbuch aus dem Jahr 2000 (vgl. Kirchenleitung 2000).

Parallel zum Ausbau der Agendenwerke entsteht im 19. Jahrhundert ein rhetorikkritisches bis rhetorikfeindliches Milieu in der evangelischen Theologie. Der Tübinger Theologe Christian Palmer etwa baut den Hauptteil seiner Homiletik von 1842 noch nach dem Vorbild der Entwicklungsstadien der Rede auf (vgl. Knape 2013:18), kritisiert aber schon hart „die Schulregeln“ (Palmer 1842:438) doktrinärer Rhetorik(ausbildung) hin zu einer ,ars bene dicendi“ (Knape 2010:30). Im Rückgriff auf eine Randnotiz Immanuel Kants - „er habe auch während einer Kanzelrede das unangenehme Gefühl, man wolle ihn überreden" (Palmer 1845:428) wendet sich Palmer nun auch von einer Rhetorik ab, die sich als ,ars persuadendi“ (Knape 2010:30) versteht. Die Wirksamkeit sieht er nicht mehr in der Form, sondern einzig im Inhalt der Predigt selbst, im Evangelium, das „von selber, wie es in Schrift und Rede an mich kommt“ wirke, ,aber deine wohlgeformten Beweise, deine rhetorischen Figuren, deine Aufzählung von Triebfedern zu gutem Handeln, - die thun's nicht“" (Palmer 1845:429, im Original mit Hervorhebungen). Ein Jahrhundert später formuliert Eduard Thurneysen die Kritik an der Beredsamkeit, die für eine ganze Strömung innerhalb der evangelischen Theologie, die sogenannte Dialektische oder Wort-Gottes-Theologie steht. Die Rolle des Predigers schließe rhetorisches Handeln von vornherein aus, sei er doch „Zeuge vor Gericht, der eine Aussage macht (was sollen da alle rednerischen Künste!), er redet nicht aus eigenem Antriebe, sondern in Auftrag; wo aber Auftrag ist, da ist eigener Antrieb Nebensache und wirkt nur verdunkelnd,. wenn er sich hervordrängt" (Thurneysen 1971:112). Letztlich sei es einzig Gott selbst, der über Gott sprechen könne, in keinem Fall der Mensch. Seit den 1970er Jahren ist eine gewisse Renaissance der rhetorischen Deutung der Predigt durch Theologen und Theologinnen zu verzeichnen. So greifen etwa Gert Otto und Albrecht Grözinger auf klassische Rhetorikmodelle zurück (vgl. z.B. Otto 1976, 1986, Grözinger 2007), während jüngst Wilhelm Gräb einen eigenständigen theologisch-rhetorischen Deutungsansatz mit Anleihen an der sprechwissenschaftlichen Kommunikationstheorie Hellmut Geißners präsentierte. Gräb will dabei die Predigt streng von der Liturgie getrennt untersucht wissen, indem er die (rhetorische) Frage stellt, ob ,die kirchliche Predigt nicht viel zu sehr zum geradezu zwanghaft verordneten Element kirchlicher Rituale geworden“" (Gräb 2013:7f.) sei.

In Linguistik und Sprechwissenschaft schließlich ist die empirische Betrachtung von Gottesdiensten nur sporadisch zum eigenen Gegenstand avanciert. Mit dem Niedergang akademischer Sprechwissenschaft nach der Zeit des Nationalsozialismus verschwindet auch dieses vorher durchaus in Ansätzen beforschte Thema. So entwickelte der Germanist Ewald Geißler ein Konzept von der „Sprechkunst des Heiligen“ (Geißler 1925:189), das der Sprechwissenschaftler Fritz Schweinsberg (1946:10) in praktischer Absicht und römisch-katholischer 
Perspektive weiterentwickelte. Die stärker empirisch orientierte Forschung in der DDR konnte den Gottesdienst aus ideologischen Gründen nie als Gegenstand gewinnen, die Landauer Sprechwissenschaft und die sich später formierende Tübinger Rhetorikschule in der BRD waren bei der Betrachtung von Predigt und Gottesdienst nicht an Empirie interessiert. Die Haltung dieser Rhetorikforschung zur Predigt manifestiert sich vielmehr als gelehrte Kritik und Ringen um ein grundsätzliches Rhetorikverständnis, etwa durch Hellmut Geißner (1977:49) und Joachim Knape (2010:31). Daran knüpfen aktuell einzelne Praxishilfen an, etwa die des theologisch-sprechwissenschaftlichen Grenzgängers Michael Thiele (vgl. z.B. Thiele 2004:240). Von Interesse für die Forschung am evangelischen Gottesdienst ist schließlich eine interaktionslinguistische Studie von Heiko Hausendorf und Reinhold Schmitt (Hausendorf/Schmitt 2014) ${ }^{1}$, die unter Rückgriff auf gesprächsforscherische Methoden die multimodale Kommunikation in einer Sonderform des evangelischen Gottesdienstes beleuchtet.

Die eigentliche Deutungsmacht über das Verständnis des evangelischen Gottesdienstes teilen sich im 20. Jahrhundert Ritualtheorie, Theater- bzw. Performancetheorie und verschiedene semiotische Ansätze, die in unterschiedlicher Tiefe theologisch rezipiert und teilweise adaptiert wurden. Durch die Aufnahme von Erkenntnissen der Rezeptionsforschung entstanden etwa in jüngeren Jahren als ein empirischer Forschungsansatz am Gottesdienst, die Heidelberger Studien zur Predigtrezeption von Helmut Schwier und Sieghard Gall, deren ,[w]esentliches Merkmal [...] die Erfassung und Analyse der ablaufsimultanen Rückmeldungen im Kontext zum Gesamteindruck, zu Details der Rezeption und zum persönlichen Hintergrund, zu Einstellungen und Erfahrungen" (Schwier 2008:8) mittels eines von den RezipientInnen bedienten Messinstruments ist. Dieser Ansatz ist indes nicht an der konkreten Sprachgestalt, sondern vielmehr an der Wirkung der Predigt interessiert. Andere Forschungsansätze verhalfen zur Deutung des Gottesdienstes als Ritual oder Ritus (zur Debatte um die Begrifflichkeit siehe Klie 2009:97 und Grethlein 2012:168f.) und verorten sich dabei innerhalb verschiedener kulturwissenschaftlicher Paradigmen, der Theater- und Performancetheorie z.B. Gräb (1997:212) und Bieritz (1998), zuletzt prominent dargestellt von Friedrich (2001) und Roth (2006); eine eigene theaterwissenschaftliche Perspektive auf Gottesdienste entwickelt (Fischer-Lichte 2006:19), in semiotischer (Bieritz 2004) oder ästhetischer Perspektive (zuerst Martin 1984). Eine empirische Betrachtung gottesdienstlichen Sprechens, seiner rhetorischen und phonetischen Merkmale, wie sie hier vorgelegt wird, sollte daran anschlussfähig sein. Einen vielversprechenden Ansatzpunkt für rhetorische Forschung mit empirischen Mitteln bietet dabei die Ritualforschung.

${ }^{1}$ Den Hinweis auf diese Studie verdanke ich Cordula Schwarze. 


\subsection{Zweiter Zugang: Rhetorikforschung als Zugang zum Persuasionsbegriff}

Hellmut Geißner profiliert gegen ein rhetorisches Modell der Rede als „Instrument eines eloquenten Situationsmächtigen" (Geißner 1977:49) die rhetorische Kommunikation, deren Ziel es sei, in gedanklicher Partizipation die Handlungsziele der redenden Person mit zu vollziehen. Er betrachtet den Gottesdienst als Gesamtvollzug, innerhalb dessen die Herstellung von Konsensgefühl und Sachkonsens sowie die gemeinsame Entscheidungsfindung als Ziele anzunehmen seien (Geißner 1977:50). Den Persuasionsbegriff wendet Geißner ausschließlich als Kontrastpol zum Informationsbegriff und ausschließlich auf die Predigt an. Hier stimmt er mit Joachim Knape überein, dem zu Folge die Predigt in Form der Textpredigt vornehmlich ihr Informations- und in Form der Themenpredigt vornehmlich ihr Persuasionspotential entfalte (Knape 2010:32). Diese Feststellung bezieht sich allerdings ausdrücklich nur auf diesen einen Teil des Gottesdienstes, die Predigt eben, sie bleibt bei Geißner wie Knape Vermutung und führt für die hier anzustellende Betrachtung nicht weiter. Persuasion ist, so formuliert es Joachim Knape unabhängig vom Kontext gottesdienstlicher Kommunikation, die „Einschränkung des Freiheitsspielraums beim Kommunikationspartner“ (Knape 2003:888). Er versteht Persuasion als den ,gesamten vom Kommunikator initiierten Überzeugungsvorgang mit dem Ziel, bei anderen einen Standpunktwechsel herbeizuführen“ (Knape 2003:874). Die persuasive Kommunikation geschehe ausdrücklich gegen Widerstände oder unter Umgehung derselben. Hierbei könne jeder beliebige mentale Zustand als Standpunkt verstanden werden. Diese Definition geht über die Übernahme reiner Vernunftwahrheiten weit hinaus. Sie ist damit auch für Glaubenskommunikation und die damit verbundenen Überzeugungen anschlussfähig. Es soll im Weiteren gezeigt werden, wie dieser Persuasionsbegriff zur Analyse evangelischer Gottesdienste dienlich sein kann.

Eine Betrachtung des Gottesdienstes auf der Spur seines persuasiven Moments muss nun dreierlei vereinen: Sie muss die besonderen Inhalte innerhalb der Gesamtgestalt des Gottesdienstes würdigen, muss dabei reale sprachliche und sprecherische Vollzüge empirisch so detailliert wie möglich wahrnehmen und sie dann anhand eines adäquaten Persuasionskonzepts analysieren.

\subsection{Dritter Zugang: Die Gestalt des Gottesdienstes als Ritual}

Um das persuasive Moment des evangelischen Gottesdienstes freizulegen, empfiehlt sich ein Zugang über Erkenntnisse der Ritualtheorie. Hilfreich ist dabei die Theoriebildung von Charlotte Humphrey und James Laidlaw (Humphrey/Laidlaw 1994). Am Beispiel eines morgendlichen Gottesdienstes des hauptsächlich in Indien verorteten Jainismus entwickeln sie die konstituierenden Merkmale 
des Rituals. Ein Ritual sei aus verschiedenen Perspektiven als „,nicht-intentional', ,vorgeschrieben', ,elementar' bzw. ,archetypisch' und schliesslich ,wieder-erkennbar"“ (Humphrey/Laidlaw 2008:135; die folgenden Ausführungen beziehen sich auf die leicht gekürzte, deutsche Übersetzung Humphrey/Laidlaw 2008:135ff.) zu beschreiben. Die Nichtintentionalität beziehe sich dabei auf das Fehlen eines intentionalen Bezugs auf ein einzelnes (sprachliches oder nichtsprachliches) Handlungssegment. Die Gesamthandlung, das Ausführen des Rituals selbst, bleibe davon unberührt und müsse sogar intentional ergriffen werden. Die Wahl der Handlungsmittel sei insofern vorgeschrieben, als sie sich nicht auf intentionales Verstehen, sondern auf ein Präskript beziehe - das Ritual müsse in einer bestimmten Weise ausgeführt werden und unterliege nicht der individuellen Gestaltungsfreiheit. Dass das Ritual nicht aufgrund der Einzelsegmente der Handlung, sondern aufgrund seiner Gesamtgestalt identifizierbar, also wiedererkennbar werde, mache seinen elementaren Charakter aus.

Die hier knapp skizzierte Charakteristik des Rituals nötigt dazu, den evangelischen Gottesdienst auch als eine Abfolge von Handlungsgestalten zu verstehen, die in ihrer Gesamtheit den Gottesdienst bilden. Abfolge und Elemente des evangelischen Gottesdienstes, die auch im zweiten Teil dieses Beitrags eine Rolle spielen, sollen nun kurz dargestellt werden.

Das Evangelische Gottesdienstbuch, das hier als ein prominentes Paradigma näher betrachtet werden soll, kennt vier Strukturteile des Gottesdienstes: „Eröffnung und Anrufung“, „Verkündigung und Bekenntnis“, „Abendmahl“" und „Sendung und Segen“ (Kirchenleitung 2000:32-35). Da die in sich selbst sehr komplexe Abendmahlshandlung einer eigenen, aufwändig zu differenzierenden Betrachtung bedürfte, wird dieser Teil hier nicht eigens behandelt.

Das Gebet kann sowohl metaphorisch als „Dialog mit Gott“ (Meyer-Blanck 2012:117), als auch als Dialog der Gemeindemitglieder untereinander (vgl. Meyer-Blanck 2012:117f.) verstanden werden. Gebete erscheinen als Bitte und Dank oder als Selbstvergewisserung der Betenden (vgl. Meyer-Blanck 2012:124). Vater-Unser-Gebet und Psalmgebet stellen Sonderformen des Gebets dar, da sie üblicherweise von Gemeinde und Liturg/-in gemeinsam auswendig und chorisch gesprochen oder abgelesen werden.

Das Glaubensbekenntnis steht der äußeren Form nach dem Vater-Unser-Gebet nahe, ist aber der Intention nach keine Bitte und kein Dank, sondern vielmehr eine Form der Selbstvergewisserung.

In der Regel treten an verschiedenen Stellen im Gottesdienst Lesungen auf, zu Beginn des Teils „Verkündigung und Bekenntnis“ und als Verlesung des Predigttextes innerhalb der Predigt. Die Lesungstexte sind vorgegeben und wiederholen sich in einem mehrjährigen Rhythmus.

Die Predigt ist eine eigene Redegattung, die auf unterschiedliche Weise, mehr oder weniger modellgeleitet gestaltet sein kann. Üblicherweise hat sie einen Predigttext zur Grundlage, der im Rahmen der Predigt gelesen wird. Die Predigt 
wird in der Regel von dem Liturgen/der Liturgin vollständig ausformuliert und abgelesen, kann aber auch auf Grundlage eines Stichwortzettels oder ganz ohne Vorlage gehalten werden.

Abkündigungen sind aktuelle Bekanntmachungen für die Kirchengemeinde bzw. die Nennung von Personen und Ereignissen, die in die Fürbitte der Gemeinde aufgenommen werden. Üblicherweise liegt dabei ein Stichwortkonzept, bzw. eine Liste mit Terminen zu Grunde. Abkündigungen können in besonderer Weise zum Handeln - zum Besuch von Veranstaltungen, zum Spenden etc. - motivieren.

Ferner lassen sich verschiedene Arten von Moderation und liturgischer Rahmung feststellen. Teile, die die Aufmerksamkeit dezidiert auf das voranliegende oder kommende Stück lenken stehen neben solchen, die monologisch oder responsorisch zwischen Gemeinde und Liturg/-in bzw. Lektor/-in vermitteln, z. B. als Gruß oder Segenswunsch. Beide Formen können hochgradig formalisiert auf Grundlage eines Präskripts auftreten (dann sowohl gesungen als auch gesprochen) und in freier, auch spontaner Formulierung (ausschließlich gesprochen). Thomas Klie unterscheidet zwischen Kommunikation mit dreistelligem Muster der salutatio, also der Vermittlung zwischen Absender/-in, Adressat/-in und Gott, und dem zweistelligen Muster der captatio, der Vermittlung zwischen Absender/-in und Adressat/-in (Klie 2010:36). Für Ulla Kloß ist das Ziel der Moderation die harmonische Verbindung der liturgischen Elemente (Kloß 2002:16) - damit selbst aber nicht zu diesen Elementen zu rechnen. Daneben gibt es weitere Formen, die der Funktion nach als Moderation erscheinen, jedoch einen eigenen inhaltlich-verkündenden Charakter aufweisen.

Predigt, Abendmahl und Gottesdienst enden in der Regel jeweils mit einem Segen. Der sog. Kanzelsegen nach der Predigt wird, wie auch die Sendung nach dem Abendmahl, gesprochen, der Segen am Ende des Gottesdienstes kann gesungen oder gesprochen vorkommen. Er kann auswendig gesprochen bzw. gesungen oder abgelesen werden.

In Bezug auf die Frage nach dem persuasiven Moment des evangelischen Gottesdienstes ergibt sich nun folgende Heuristik: Der inhärente Zweck des Rituals bezieht sich auf das Ritual als solches selbst. Damit ist es der freien Wahl der Gestaltungsform unter den Bedingungen rhetorischer Zweck-Mittel-Reflexion entzogen. Dies gilt für den Gottesdienst als Ganzen. Von dieser ritualhaften Gesamtgestalt des Gottesdienstes ist die Gestalt seiner einzelnen Teilelemente zu unterscheiden. Gänzlich ritualisierte Elemente können kein rhetorisches, mithin kein persuasives Potential haben, da sie ja selbstreferentiell sind. Finden sich aber im Gottesdienst solche Handlungsgestalten, die nur eingeschränkt oder gar nicht ritualisiert sind, unterliegen sie der individuellen rhetorischen und ästhetischen Mittelwahl. Rhetorische Elemente sind fernerhin darauf zu überprüfen, ob sie aufgrund ihrer argumentativen Struktur als persuasiv verstanden werden können, ob sie also dazu gestaltet und dazu geeignet sind, einen Wechsel des mentalen Zustandes der Rezipientinnen und Rezipienten zu initiieren. Diese Heuristik bildet die Grundlage für die Unter- 
suchung des persuasiven Potentials des evangelischen Gottesdienstes, wie sie im Folgenden durchgeführt wird.

\section{Zur Empirie des persuasiven Moments des evangelischen Gottesdienstes}

Nachdem gezeigt wurde, unter welchen Bedingungen dem evangelischen Gottesdienst persuasives Potential beigemessen werden kann, soll die Wirkweise von Persuasion nun anhand von Beispielen illustriert und analysiert werden. Zuerst werden die expliziten Forschungsfragen benannt und das Datenmaterial sowie die angewendeten Erhebungsmethoden dargestellt. Am Argumentationsgang einer Abkündigung wird dann die argumentative Mikrostruktur, am Argumentationsgang innerhalb eines gesamten Gottesdienstes die argumentative Makrostruktur dargestellt.

\subsection{Forschungsfragen}

Um sich realen Gottesdiensten in angemessener Weise zu nähern, sollen zunächst konkrete Forschungsfragen aufgestellt werden. Da das Ziel dieses Beitrags der Nachweis persuasiver Strategien in evangelischen Gottesdiensten ist, müssen diese am Beispiel nachgewiesen werden und ihrer Form und Funktion nach bestimmt werden. Sie werden folgendermaßen formuliert und überprüft:

1. Lassen sich in der Gesamtgestalt von evangelischen Gottesdiensten persuasive Strategien nachweisen?

Der Nachweis geschieht durch die Darbietung von Transkripten, die eine rhetorische Streitfrage beinhalten, die kommunikativ bearbeitet wird.

2. Durch welche sprachlichen und sprecherischen Formen ist die Argumentation bestimmt?

Die Beantwortung dieser Frage geschieht durch die Darstellung der erhobenen Daten auf sprachlicher uns sprecherischer Ebene.

3. Welche Funktion kann hinter dem Einsatz sprachlicher und sprecherischer Mittel mit dem Zweck der Persuasion vermutet werden?

Die Beantwortung dieser Frage geschieht im Rückgriff auf die Analyse vergleichbarer Kommunikationssituationen, wie sie in der rhetorischen und sprechwissenschaftlichen Fachliteratur zu finden sind.

4. Durch welche rhetorische(n) Strategie(n) ist Argumentation in Gottesdiensten bestimmt?

Die Beantwortung dieser Frage geschieht durch die abschließende und auswertende Analyse der Transkripte. 
Dieser Beitrag stellt eine qualitative, explorative Studie dar, die sich zur Beschreibung einzelner Phänomene auch quantitativ ausgewerteter Daten bedient. Dabei steht die Präsentation des erhobenen Datenmaterials im Zentrum, nicht die Validierung übergreifender Hypothesen.

Die folgenden Ausführungen und methodischen Entscheidungen knüpfen an eine explorative empirische Studie (Schulz 2014) an. Innerhalb dieser Studie wurden theologische und die rhetorisch-sprechwissenschaftliche Perspektiven auf gesprochene Sprache in evangelischen Gottesdiensten dargestellt. Darüber hinaus wurden erste Daten zu evangelischen Gottesdiensten erhoben und kommentiert. Grundlage des empirischen Teils waren drei Gottesdienste aus verschiedenen Bekenntnistraditionen der Evangelischen Kirche in Deutschland, die auf etwaige Muster hinsichtlich der Merkmale Artikulationsgeschwindigkeit, Pausierung und Betonung hin untersucht wurden.

Dieser Beitrag bedient sich der Rohdaten dieser Studie, setzt dabei aber einen konkreten Fokus auf Phänomene der Persuasion.

\subsection{Daten und Methoden}

\subsubsection{Datengewinnung und -aufarbeitung}

Bei der Datengewinnung war zwischen Zugänglichkeit und Natürlichkeit abzuwägen. Radio- und Fernsehgottesdienste schieden aus, da ihnen in der Regel eine besondere Vorbereitung und ein Coaching der Beteiligten voraus geht. Die selbständige Gewinnung qualitativ hochwertiger Aufnahmen wäre aufgrund der in Kirchenräumen üblichen Hallphänomene äußerst aufwändig geworden und hätte voraussichtlich die beobachtete Situation in zu hohem Maße, etwa durch Aufstellen zusätzlicher Richtmikrophone, beeinträchtigt. Ein Mittelweg verband zu einem gewissen Grad die Vorteile beider Erhebungsansätze. Damit etwa ,[ä]ltere Menschen, die den Gottesdienst am Sonntag aus gesundheitlichen Gründen nicht mit feiern können, [...] die Möglichkeit [haben], den Gottesdienst auf Kassette zu Hause zu hören" (IQ 1) nehmen viele Kirchengemeinden ihre Gottesdienste gewohnheitsmäßig auf. Einige davon stellen die Aufnahmen im Anschluss an den Gottesdienst zur freien Verfügung ins Internet. Eine Auswahl dieser Gottesdienste wurden kursorisch durchgehört und ein Teil davon in Gänze transkribiert. Daten aus zwei Gottesdiensten kommen nun hier zur Präsentation. Mit der Einschränkung, dass auf Ganze gesehen relativ wenige Kirchengemeinden ihre Gottesdienste auf diese Weise zugänglich machen und damit eine beschränkte Auswahl zur Verfügung steht, stellt sich dennoch eine gute Datengrundlage dar.

In diesem Beitrag sollen nun Ausschnitte aus zwei Gottesdiensten dargestellt werden. Der erste Gottesdienst wurde am 16. März 2014 in der ostfriesischen Kirchengemeinde Loga gehalten, der zweite am 9. Februar 2014 im westfälischen 
Iserlohn. Die genaue Einordnung der Gottesdienste in Bekenntnistradition und Kirchenjahr spielt für die weiteren Ausführungen keine Rolle (vgl. dazu Schulz 2014:42, $55,67)$.

\subsubsection{Analysemethoden}

Um die Leitfragen dieses Beitrags, besonders im Hinblick auf die Funktion sprachlicher und sprecherischer Merkmale, angemessen zu bearbeiten, ist es nötig, bewährte Analysemethoden einzusetzen. Damit wird eine Verankerung im Fachdiskurs möglich und es erscheinen Ansatzpunkte für die weitere Bearbeitung des Gottesdienstes als Themenfeld für die rhetorische Forschung.

Die sprachlich-rhetorische Analyse schließt an die Forschung zur Beweisund Argumentationslehre an, wie sie bei Aristoteles (2002) grundgelegt und in jüngster Zeit etwa bei Atayan (2006) und Schwarze (2010) aktualisiert wurde. Dabei werden Erkenntnisse aus der Rede- und Gesprächsforschung auf das Datenmaterial angewendet.

Die sprecherische Analyse schließt an die Gesprochene-Sprache-Forschung an, wie sie in Einzeluntersuchungen etwa von Uhmann $(1992,1996)$ und in übergreifender Zusammenstellung bei Schwitalla (2012) vertreten wird. Zentrale Aufmerksamkeit liegt hier auf dem Rhythmus als Merkmal gesprochener Sprache der in der Zusammenschau aus Artikulationsgeschwindigkeit, Pausierung und Betonung bzw. Akzentuierung erkenn- und bestimmbar wird. Um die Aufnahmen einer eingehenden Analyse zugänglich zu machen, wurden zunächst die vollständigen Gottesdienste mit dem Programm f4 von dr. dresing \& pehl GmbH (IQ 2) nach der Konvention GAT-2 (Selting et al. 2009) transkribiert. Grundlage bildet das Basistranskript mit Betonungen, in das gemessene Pausen und Artikulationsgeschwindigkeit für jede Phrase eingetragen wurden. Zwei unabhängige Sprechwissenschaftler haben die Fokusakzente ausgewählter Passagen zur Kontrolle abgehört und den Befund bestätigt. Die Pausen wurden mit PRAAT (IQ 3 ) mit Hilfe eines angepassten Scripts (IQ 4) festgelegt und ausgemessen. In Aufnahme der allgemein verbreitetsten Kategorie (vgl. etwa O'Connell/Kowal 2008:50 -53, zum „fehlenden Konsens“ in dieser Frage Pfitzinger 2001:123-125) wird das Sprechtempo als Artikulationsgeschwindigkeit (vgl. Kowal 1991:27) angegeben. Pausen werden hier in Anschluss an Kowal (1991:35) als „Mindestdauer der Artikulationsabwesenheit" von mindestens 0,27 Sekunden definiert.

Auf Grundlage der Daten wäre eine Ausweitung auf andere Merkmale grundsätzlich möglich und erstrebenswert und für eine weitere Vertiefung der Erkenntnisse gewinnbringend, wurde hier aber nicht erwogen, um den Rahmen dieses Beitrags zu wahren. Zu denken wäre dabei an eine umfassende Abbildung sprechmelodischer Merkmale wie etwa der Sprechstimmlage oder von Intonationsverläufen. Für eine umfassendere Darstellung des rhetorischen Gesamtvollzugs wären ferner sprachbegleitende Gesten oder proxemische Verhältnisse im 
Raum interessant. Sie sind auf Grundlage der reinen Audiodaten jedoch schlechterdings nicht darzustellen.

\subsection{Persuasion in evangelischen Gottesdiensten, Mikro- und Makrostuktur}

An zwei Beispielen soll nun das persuasive Moment des Gottesdienstes ausschnittartig dargestellt und untersucht werden. Dazu wurden das Vater-Unser-Gebet, das Psalmgebet und das Glaubensbekenntnis, alle strukturierenden Teile der liturgischen Rahmung, die Bibellesung und der Segen nach der Predigt und zum Abschluss des Gottesdienstes als agendarisch vorgeschriebene Texte aus der Betrachtung ausgeschieden. Die Predigt unter Einbezug der Lesung des Predigttextes, frei formulierte Gebete, die Abkündigungen und die moderierenden Teile können also auf ihr rhetorisches Potential hin untersucht werden.

Mit Vahram Atayan soll zwischen der Mikro- und der Makrostruktur von Argumentation unterschieden werden, wobei die Makrostruktur als „Konstellation [...] von kommunikativen Handlungen angesehen [...] [wird], bei deren Interpretation mindestens zwei minimale Argumentationen anzunehmen sind" (Atayan 2006:50). Im ersten Beispiel aus der Kirchengemeinde Loga versucht der Pfarrer, die Beteiligung an der gemeindlichen Gartenaktion zu erhöhen. Die Argumentation beschränkt sich dabei auf einen Teil des gottesdienstlichen Strukturelements der Abkündigungen. Im zweiten Beispiel argumentieren ein Moderator und die Pfarrerin für die These, dass der Text der Bibel existentielle Geltung für Menschen haben könne. Die Gesamtargumentation verteilt sich dabei auf verschiedene gottesdienstliche Strukturelemente.

\subsubsection{Die Gartenaktion. Mikrostruktur der gottesdienstlichen Argumentation}

Gegen Ende des Gottesdienstes der Kirchengemeinde Loga stehen, nach dem letzten Gebet und vor den Fürbitten und dem Abschlusssegen, die Abkündigungen. Sie sind durch sechs längere Pausen von je über einer Sekunde entsprechend den behandelten Themengebieten auch sprecherisch erkennbar gegliedert. Auf die längste Pause folgt ein interessantes Segment, in dem der Pfarrer (PFR) auf eine kommende Veranstaltung hinweist:

Beispiel (1): (Abkündigung zur Gartenaktion Gottesdienst Loga, 46:39-47:20)

01 PFR $\quad\left[\begin{array}{l}{[5]^{2} \text { am SONNabend dann }} \\ 02\end{array} \quad(0.9)\right.$

2 Die Artikulationsgeschwindigkeit (AG) bezieht sich im Folgenden immer auf das folgende Segment und wird in Silben pro Sekunde fett in eckigen Klammern angegeben. 


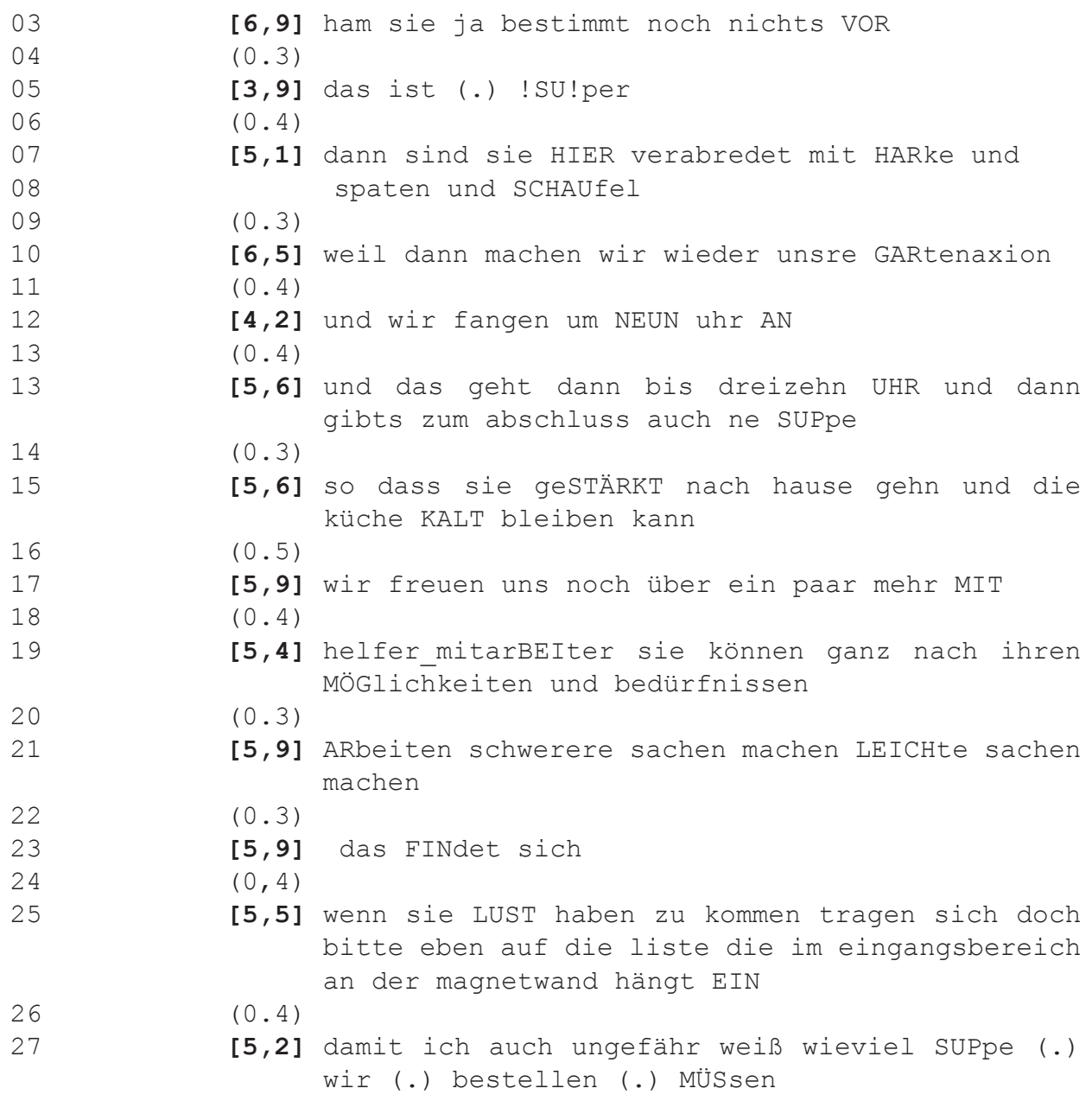

In diesem Beispiel dominieren parataktische Satzkonstruktionen. Der Pfarrer spricht in assoziativen Ketten (Zeilen 21-23) von einem ihm und vermutlich auch dem Großteil der Gemeinde bekannten Thema. Die Pausen und Betonungen folgen hier deutlich den Sinnschritten der Aussage bei einem Pausenzeitquotienten von 1,74. Liegt im Durchschnitt der gesamten Abkündigungen die Artikulationsgeschwindigkeit bei 4,9 Silben pro Sekunde, so sind es in dem präsentierten Ausschnitt 5,5. Dieser Text ist also deutlich schneller artikuliert, als seine Umgebung. Die persuasive Intentionalität der Kommunikation ist nicht zu bestreiten. Das Ziel der Ausführungen ist es, ausreichend Personen an der „Gartenaktion“ zu beteiligen (zentral formuliert in Zeilen 17-19). Der rhetorische Streitpunkt ist es also, ob man sich zu dieser Veranstaltung anmelden soll. Um eine höhere Beteiligung zu erreichen, macht der Pfarrer zwei Angebote, nämlich Zeitvertreib (Z. 01-03) und Bewirtung (Z. 13-15), die beide mit dem Angebot von Gemeinschaft (Z. 07) verbunden sind. Besonders 
auffällig ist die übermäßig starke Betonung eines Wortes (Z. 05). Durch die Emphase wird der Sprechakt des Lobens intensiviert (vgl. Schwitalla 2012:60f.), was zur Motivation der Hörerinnen und Hörer beitragen kann. Dem möglichen Einwand, der geplante Arbeitseinsatz könnte körperlich zu anstrengend sein, kommt der Pfarrer zuvor, indem er die konkrete Form der Beteiligung offen lässt (Z. 19). Auch hier verbindet er seine Argumentation mit einem positiven Angebot (Z. 19). Schließlich wird die Beteiligung nicht an die erzwungene Bereitschaft der Angesprochenen, sondern an ihre emotionale Beteiligung, die Lust, dabei zu sein (Z. 25) geknüpft. Was inhaltlich die Bitte um Beteiligung darstellt, wird formal als Veranstaltungsankündigung dargeboten. Der Pfarrer spielt hierbei mit den verschiedenen Textsorten.

Die hier beispielhaft angeführte Argumentation in einem Abschnitt der Abkündigungen ist aus verschiedenen Perspektiven interessant. Einerseits wird damit gezeigt, dass die Funktion von Persuasion in evangelischen Gottesdiensten sich auch auf ganz alltägliche Koordinierungserfordernisse beziehen kann. Ferner ist hier eine sehr spontansprachliche Sprach- und Sprechweise zu finden, die semantisch keinen Bezug zu Glaubenskommunikation und hinsichtlich Betonungsmuster und Tempo keine ritualisierten Sprechmuster aufweist, wie sie im restlichen Gottesdienst immer wieder anzutreffen sind und wie sie auch in folgendem Beispiel gefunden werden können.

\subsubsection{Die Geltung der Bibel. Makrostruktur der gottesdienstlichen Argumentation}

Gottesdienste sind nicht alleine von pragmatischen Erwägungen zur Beteiligung bei Gemeindeaktivitäten bestimmt, soviel dies ein relevanter und legitimer Teil sein mag. Ein Gottesdienst kann auch insgesamt ein Argumentations- und Überzeugungsziel verfolgen. Im Fall des untersuchten Gottesdienstes aus Iserlohn wird das besonders gut deutlich. Das Argumentationsziel des Gottesdienstes ist, knapp zusammengefasst folgende: „Die Botschaft der Bibel ist für das Leben relevant". Hier ist es nicht allein die Pfarrerin, sondern es sind verschiedene sprecherisch beteiligten Personen, die zusammen die in der Predigt gipfelnde und in ihr zentral ausgeführte Argumentation vor- und nachbereiten.

In der Begrüßungsansprache stellt ein mitwirkendes Gemeindemitglied dar, dass in der Predigt zu erörtern sein werde, dass die Botschaft von Jesus nicht nur eine Märchengeschichte, sondern Begegnung mit Jesus selbst sei. Im Schuldbekenntnis führt die Pfarrerin dann aus, dass die Erkenntnis der Wahrheit der Botschaft in der Gemeinde durch ihre schuldhafte Selbstbezüglichkeit verhindert werde und dass daraus nur Gott helfen könne. In einem Gebet stellt die Pfarrerin fest, dass die angemessene Erkenntnis bei allen Betenden wohl gegeben sei, dass sie aber dennoch der stetigen Hilfe durch Gott und des eigenen Bemühens bedürfen. In der Predigt argumentiert die Pfarrerin dann in sieben Schritten: Für viele sei die Bibel ein Märchenbuch, für manche aber mehr als das. Sie könne nämlich Menschen existentiell betreffen. Dies bezeugten die Texte der Bibel selbst, ebenso 
Menschen seit ihrer Entstehung und Zeitgenossen. Ein existentielles Verständnis der Bibel sei also auch heute möglich. In performativer Weise stellt sie durch die Sendung nach dem Abendmahl die Geltung der Texte der Bibel abschließend als gültig dar.

Da eine Zitierung des gesamten Gottesdienstes hier zu viel Raum einnehmen würde, soll die Struktur dieses Gottesdienstes kurz dargestellt und dabei die Verteilung argumentativer Bezüge und Positionen ausschnittartig dargestellt werden. Da die einzelnen Teile der Gesamtargumentation für sich gesehen wiederum geschlossene Argumentationen darstellen, kann der hier in Ausschnitten präsentierte Gottesdienst als argumentative Makrostruktur (Atayan 2006:50) aufgefasst werden.

Der Gottesdienst beginnt mit Glockengeläut und Gesang. Schon in der Begrüßung, die durch ein Mitglied der Gemeinde in der Rolle des Moderators (MOD) durchgeführt wird, wird das Argumentationsziel des Gottesdienstes erstmals benannt:

Beispiel (2): (Anmoderation Gottesdienst Iserlohn, 05:01-05:27)

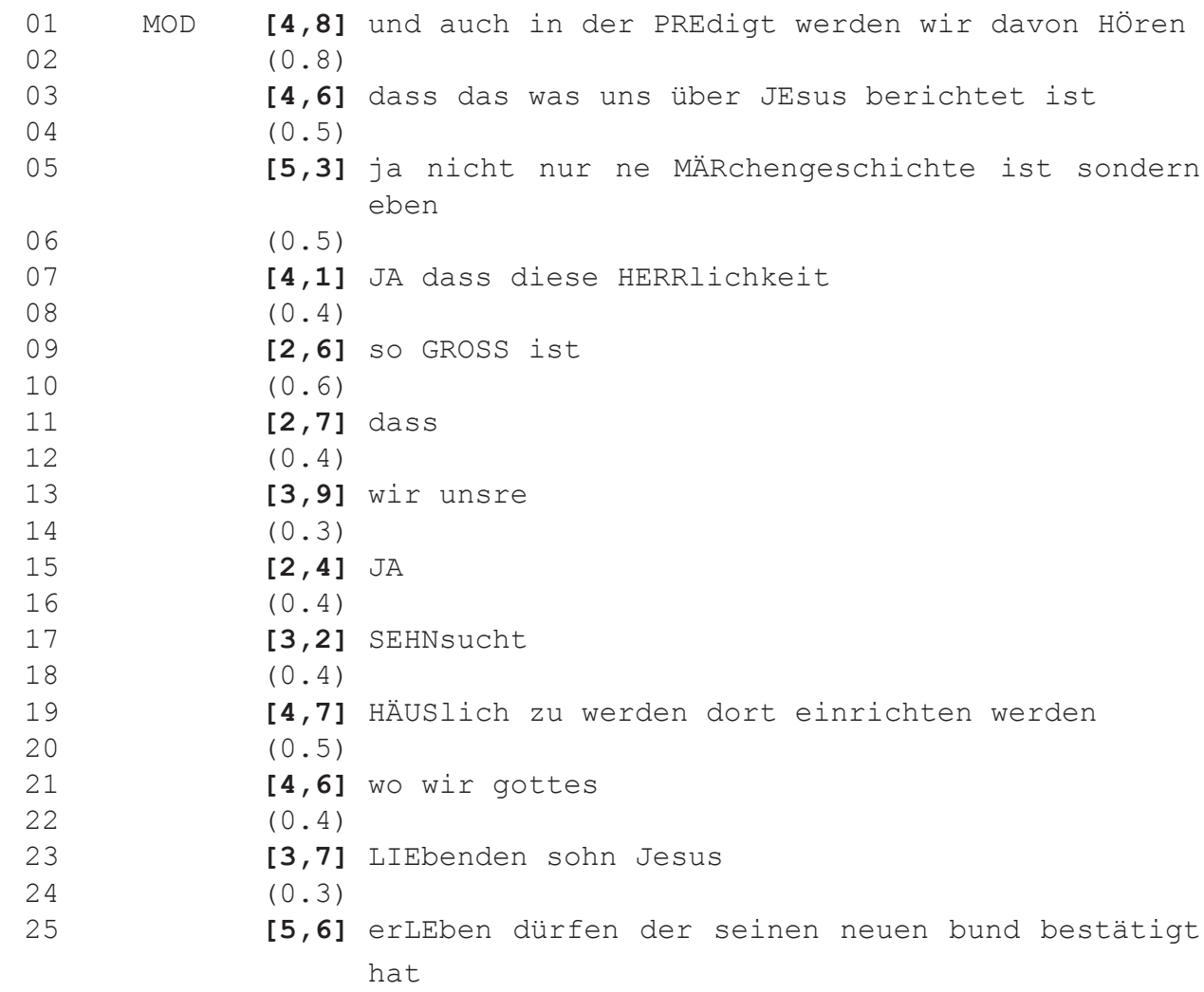


Diese Episode ist aufgrund der Hallphänomene (zu Beginn gab es Probleme mit dem Mikrophon) und aufgrund der Spontansprachlichkeit (Zögern, Häsitationspartikel in 15) von sehr vielen, gleichförmigen Pausen (zehn Mal zwischen 0,3 und 0,5 Sekunden, je einmal 0,6 und 0,8 Sekunden) unterbrochen. Der Moderator stellt als Quaestio des Gottesdienstes auf, dass als strittig gelten könne, ob die biblische Botschaft eine Märchengeschichte sei. Als Beweisziel der Predigt führt er ein, dass sie eben nicht als Märchengeschichte zu bewerten sei.

Es folgen das durch die Pfarrerin anmoderierte Psalmgebet, ein liturgischer Wechselgesang und das Schuldbekenntnis. Das Schuldbekenntnis stellt hier eine Sonderform des Gebets dar. Darin spricht die Pfarrerin (PFN) die Schuld der Gemeinde aus. Weit verbreitet ist ein formelhaftes Schuldbekenntnis (Kirchenleitung 140), in diesem Fall nimmt es aber die Pfarrerin zum Anlass, das Thema des Gottesdienstes aufzugreifen:

Beispiel (3): (Schuldbekenntnis Iserlohn, 09:06-10:31)

\begin{tabular}{|c|c|c|}
\hline PFN & {$[3,4]$} & lasst uns vor gott unsre SCHULD bekennen \\
\hline 02 & $(2.7)$ & \\
\hline 03 & {$[4,2]$} & gott VAter im HIMmel \\
\hline 4 & $(0.8)$ & \\
\hline 5 & {$[3,6]$} & dein SOHN jesus SAGT er ist das LICHT de[r w]elt \\
\hline 6 & $(0.4)$ & \\
\hline 7 & {$[3,9]$} & wir wollen das GLAUben \\
\hline 8 & $(5.3)$ & \\
\hline 9 & {$[3,6]$} & $\begin{array}{l}\text { wir WOLlen dass DEIN licht SCHEINT in unserm } \\
\text { ALLtag und in ALL unsren DUNKlen ECKen }\end{array}$ \\
\hline 0 & $(1.3)$ & \\
\hline 1 & {$[4,2]$} & aber es fällt uns oft so SCHWER dir zu vertrauen \\
\hline 2 & $(0.4)$ & \\
\hline & {$[2,0]$} & $\begin{array}{l}\text { es fällt uns SCHWER uns GANZ auf dich EINzulas- } \\
\text { sen }\end{array}$ \\
\hline & $(0.9)$ & \\
\hline & {$[3,6]$} & $\begin{array}{l}\text { es fällt uns SCHWER (.) die ANGST loszulassen } \\
\text { die uns beGLEItet wie ein sCHATten }\end{array}$ \\
\hline & $(1.0)$ & \\
\hline 7 & {$[4,7]$} & $\begin{array}{l}\text { die TRAUrigkeit loszulassen die unsren blick auf } \\
\text { alles verFINStert }\end{array}$ \\
\hline & $(1.0)$ & \\
\hline 9 & {$[4,8]$} & $\begin{array}{l}\text { den ÄRger loszulassen auf MENschen und auf LE- } \\
\text { bensumstände }\end{array}$ \\
\hline 20 & $(0.5)$ & \\
\hline - & {$[4,4]$} & $\begin{array}{l}\text { den ÄRger der unser HERZ in DÜsternis verSINken } \\
\text { lässt }\end{array}$ \\
\hline & $(1.8)$ & \\
\hline & {$[4,0]$} & $\begin{array}{l}\text { DEIN licht will bei uns SCHEInen (.) deine HERR- } \\
\text { lichkeit will bei uns AUFgehn }\end{array}$ \\
\hline
\end{tabular}




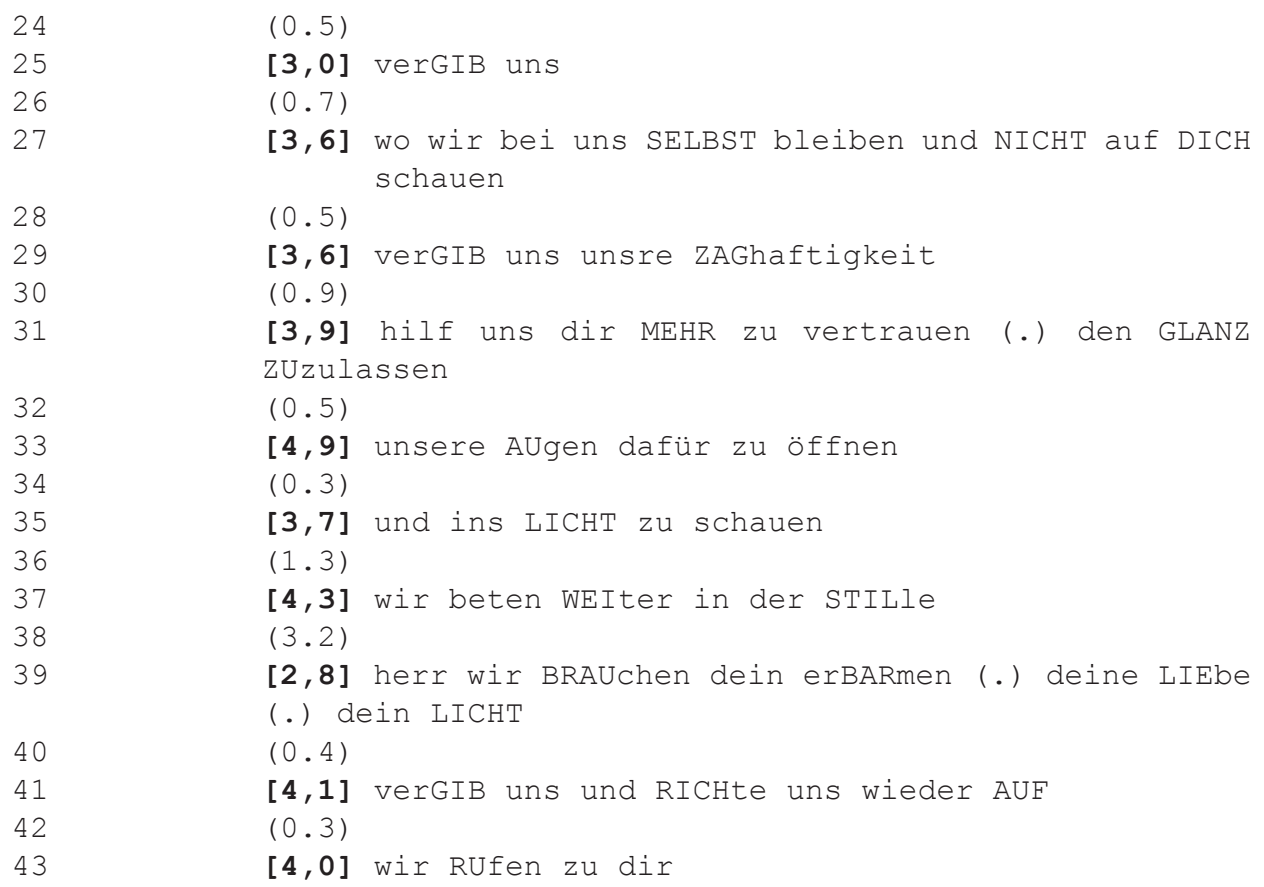

Viele der hier eingesetzten Metaphern, insbesondere die die gesamte Sequenz durchziehende Licht- und Glanzmetaphorik (Zeilen 05, 09, 15, 21, 23, 31, 35, 39) wird erst in der Predigt eindeutig auf die Bibel als Grundlage der Erkenntnis bezogen und wird hier nicht eigens motiviert. Die Gleichförmigkeit der Sprache, die ernste Gestimmtheit und die Nicht-Spontansprachlichkeit spiegeln sich in relativ hohem Pausenzeitquotienten $(1,38)$ und relativ niedriger durchschnittlicher Artikulationsgeschwindigkeit (3,8 Silben pro Sekunde). Inhaltlich entsteht der Eindruck, dass die Erkenntnis (Z. 05) nicht nur strittig, sondern die fehlende Erkenntnis geradezu ein allgemeines Merkmal der Gemeinde und der Pfarrerin, mithin des Menschen insgesamt sei (Z. 11). Die hier performativ getroffene, zugrunde liegende Annahme ist, dass der Mensch bei der Bemühung um die richtige Erkenntnis der Hilfe Gottes bedürfe (Z. 39) und dass die fehlende Erkenntnis darüber hinaus schuldhaft sei (Z. 01, 41). Absurd kurz erscheint die Pause für die Gebetsstille von 3,2 Sekunden (Z. 38). Im Widerspruch zu der inklusiven ersten Person Plural fragt die Pfarrerin die Gemeinde nach dieser zitierten Sequenz, ob das Vorgenannte das aufrichtige Bekenntnis aller sei, wechselt also die Sprecherposition und das Register in rituell-formelhafte Sprache. Nach dem gemeinsamen „Ja“ der Gemeinde spricht sie sie kraft ihres Amtes von der gemeinsam bekannten Schuld frei. Aus der Perspektive strategischer Sprachlichkeit befindet sich die Hörerschaft hier in hohem Maße in Unfreiheit, da die Oratorin ihr in einer frei gestalteten Rede Eigenschaften zuspricht (einen „Standpunkt“ im Sinne von Knape 2003:874), auf die sie nicht anders als mit dem formelhaften Inventar ritueller 
Kommunikation antworten kann. Die Hörerinnen und Hörer, die ja zugleich im rituellen Rahmen zu Sprecherinnen und Sprechern werden, haben zumindest virtuell gemeinsam einen Standpunkt eingenommen, der dem Beweisziel des Gottesdienstes entgegensteht und in der Folge Bezugspunkt der weiteren Argumentation werden kann.

Der Weg zur Veränderung des Standpunktes wird nach zwei Gemeindeliedern, aber noch weit vor der ausführlichen Argumentation in der Predigt durch ein Gebet vorweggenommen, das die Pfarrerin stellvertretend für die Gemeinde spricht:

Beispiel (4): (Gebet Iserlohn, 12:29-13:03)

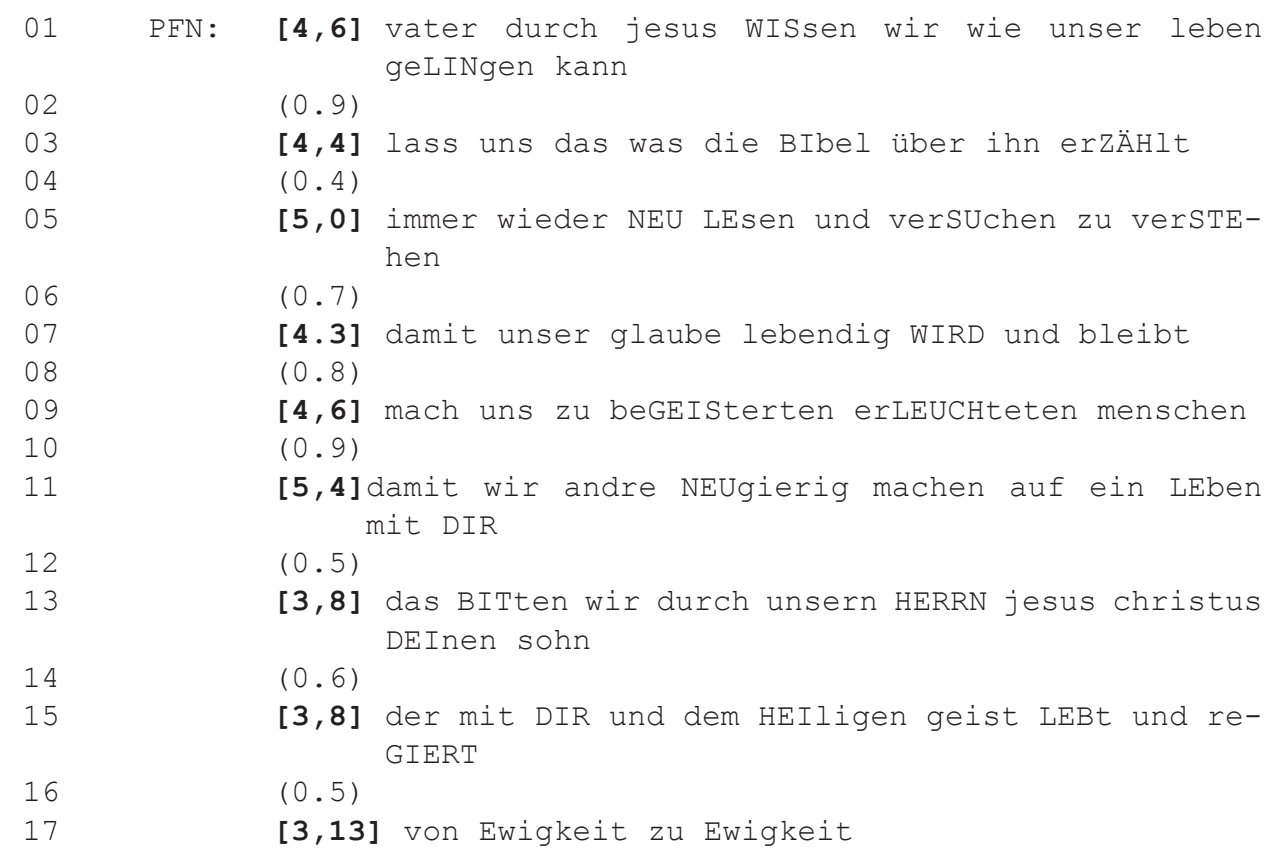

Die Pfarrerin formuliert hier mehr als eine Bitte oder einen Dank, indem sie ein gemeinsames Wissen ausdrückt, das sich aus göttlicher Offenbarung speist (Z. 01). Hier findet sich ein klassischer Topos aus der Autorität (vgl. Aristoteles 2002:1398 b20 -28). Die Pfarrerin verbindet die Bekundung dieses Wissens mit der Bitte um das richtige Verständnis dieser Botschaft (Zeilen 03, 05). Implizit argumentiert die Pfarrerin, dass das richtige Verständnis mit Gottes Hilfe auch von eigener Bemühung abhängt (Z. 05). Im Gegensatz zum vorher besprochenen Schuldbekenntnis ist dieses Element ausweislich des Pausenzeitquotienten und der Artikulationsgeschwindigkeit (4,31 Silben pro Sekunde im Durchschnitt) eher spontansprachlich geprägt. Die Betonungen sind ungewöhnlich verteilt. Nur 
eine der neun Phrasen hat eine Betonung (Z. 07), drei Phrasen zwei Betonungen (Z. 03, 17), vier Phrasen drei Betonungen (Z. 01, 09, 12, 13) und zwei Phrasen vier Betonungen (Z. 05, 15). Besonders Phrase 05 ist auffällig, da durch die Betonungshäufung kein eindeutiger Fokus mehr auszumachen ist. Die Verbindung mit hoher Artikulationsgeschwindigkeit (5,0 Silben pro Sekunde) lässt auf ikonische Sprachverwendung schließen (Schwitalla 2012:74, Uhmann 1992:330). Die Sprechform bildet hierbei das wiederholte Lesen und den Versuch des Verstehens ab. Zugleich wird der frei formulierte Gebetstext rhythmisch an den agendarisch vorgegebenen Schluss (Zeilen 13-17) angeglichen. Freie Formulierung wird hier also als rituelle Kommunikation eingefärbt. Der Übergang von selbst formuliertem Gebet zu rituellem Gebetsschluss wird durch ein Ansteigen der Artikulationsgeschwindigkeit auf 5,4 Silben pro Sekunde (Z. 13) und anschließendem Abfall auf 3,8 Silben pro Sekunde (Z. 15, 17) markiert. Agendarische Texte, etwa rituelle Formeln, sind das Gegenteil von individueller Gestaltung. Mit der rituellen Färbung frei formulierter Texte geht Geltungsgewinn einher. Die frei formulierten Texte erhalten dabei Anteil an der Geltung der von Autoritäten ratifizierten rituellen Texte. Argumentationstheoretisch kann hier der Einsatz des Topos aus der Autorität (vgl. Aristoteles 2002:1398 b20-1399 a7) beobachtet werden. Die damit verbundene „soziale Positionierungsaktivität“ (Schwarze 2010:68f.) der Pfarrerin gegenüber der Institution Kirche als normgebender Institution erfolgt hier jedoch implizit durch Übernahme des Sprechmusters der Betonungshäufung, die als Sprechform agendarischer Texte bekannt ist.

Auf das Gebet folgen die Anmoderation des Kindergottesdienstes, ein weiteres Gebet, die Lesung eines biblischen Textes, das Glaubensbekenntnis und noch ein Lied. Die anschließende Predigt ist insgesamt als Argumentation aufgebaut. Um den Argumentationsgang zu verdeutlichen, seien einzelne Etappen knapp zitiert und hinsichtlich ihres persuasiven Potentials ausgewertet. Der Fokus der Analyse liegt auf der sprachlichen Argumentation, aufgrund der Kürze mancher Ausschnitte beschränken sich die Hinweise auf die sprecherische Gestaltung auf besondere Auffälligkeiten.

Die Pfarrerin steigt ein, indem sie eine ihrer Darstellung nach allgemein vertretene Position referiert, dass nämlich biblische Texte über ihre Lesart als Märchen hinaus keine Geltung haben:

Beispiel (5): (Predigt Iserlohn Ausschnitt I, 24:46-25:07)

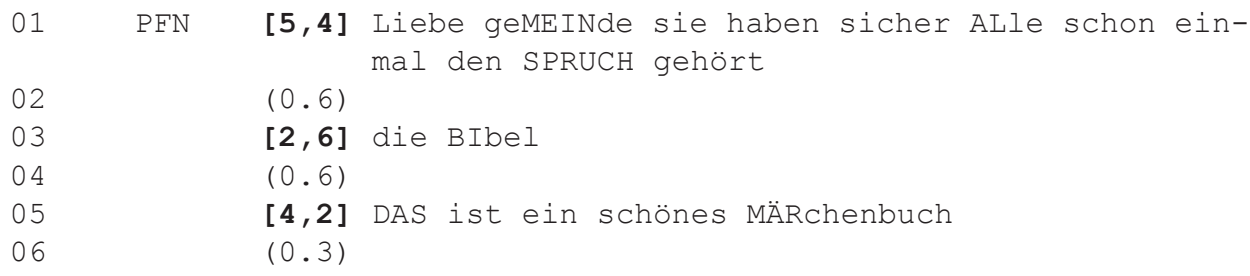


07

08

09

10

11

12

13

14

15

16

17
$[4,6]$ da kann man nett drin Lesen

$(0.8)$

$[5,5]$ aber es ist eben ein MÄRchenbuch

$(1.7)$

$[3,9]$ wer das SAGT meint EIgentlich

$(0.4)$

$[3,8]$ SCHÖne geschICHten

$(0.4)$

$[4,1]$ NETte AUSsagen

$(0.4)$

$[3,8]$ aber mit MIR hat das NICHTS zu TUN

Der Anfang der Predigt ist durch den Wechsel von Sprechhaltungen geprägt. Direkt auf die Anrede folgt eine Zitation mit Paraphrase. Artikulationsgeschwindigkeit und Pausenlänge schwanken stark und vermitteln so den Eindruck spontansprachlich direkter Ansprache. Der lokale Einstieg in die Argumentation geschieht wieder über einen Topos aus der Autorität. Von der zitierten Meinung könne man annehmen, dass ,alle und immer (so urteilen), wenn nicht das, dann aber die meisten" (Aristoteles 2002:1398b 21-23). Die Betonungshäufungen in den Zeilen 11-17 markieren die Zitation und gleichzeitig eine Distanzierung vom Zitat (vgl. Schwitalla 2012:78).

Einige Sätze später etabliert die Pfarrerin die Gegenthese, dass biblische Texte für einige Personen offensichtlich doch eine Geltung besitzen, die über die eines Märchenbuchs hinausgeht.

Beispiel (6): (Predigt Iserlohn Ausschnitt II, 25:42-25:53)

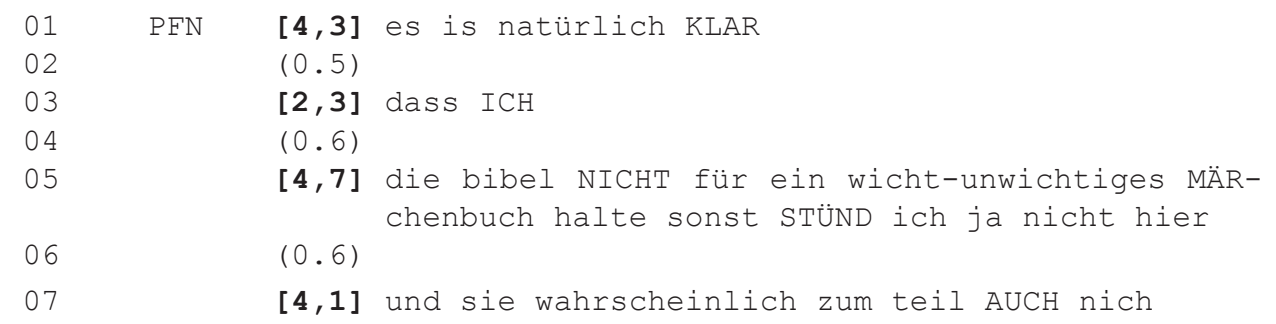

Als Beleg führt sie sich selbst an und eröffnet für die Hörerinnen und Hörer die Möglichkeit, sich mit ihr zu identifizieren, bzw. anzunehmen, dass eine entsprechende Haltung von weiteren Hörerinnen und Hörern geteilt werde. Dazu bedient sie sich des Topos aus der Person, genauer aus ihrer Art der Betätigung (Z. 05) (vgl. Schwarze 2010:71), ist sie als Pfarrerin doch gleichsam durch ihre affirmative Haltung zur biblischen Botschaft definiert. Im Fokus steht nun dieser Standpunkt der Pfarrerin und - mit Einschränkungen (Z. 07) - der versammelten Gottesdienstgemeinde. Diese Einschränkung ist insofern für die Argumentation relevant, als sie an das Schuldbekenntnis und das Gebet anknüpft. Die Pfarrerin 
lässt weiter die Option bestehen, dass es unter den Angesprochenen Personen gibt, die mit den folgenden Ausführungen überzeugt werden könnten. Gleichzeitig ermöglicht sie es dem oder der Einzelnen, sich bereits jetzt auf ihre Seite der Argumentation zu stellen. Diese Funktion wird auch durch gehäufte Auslautelisionen (Zeilen 01, 05, 07) befördert. Die Pfarrerin wechselt hier das Register im Vergleich zur Eingangssequenz der Predigt und stellt dadurch eine größere Nähe zur Gottesdienstgemeinde her (Schwitalla 2012:50f.). Zusammen mit der Korrektur (Z. 05), der doppelten Einschränkung (Z. 07) und der Variation der Artikulationsgeschwindigkeit wird so der Eindruck von Spontansprachlichkeit gefestigt. Die Pfarrerin stellt ihre persönliche Haltung gegen die eingangs von ihr zitierte, unpersönlich-allgemeine Haltung. Sie bedient damit die rhetorische Ebene des Ethos (vgl. Aristoteles 2002:1356a 5f.). All dies zusammen soll zur Erhöhung der Glaubwürdigkeit der Oratorin beitragen.

Im direkten Anschluss fragt die Pfarrerin nach Möglichkeiten des Beweises.

Beispiel (7): (Predigt Iserlohn Ausschnitt III, 25:54-26:12)

\begin{tabular}{|c|c|c|}
\hline PEN & {$[4,8]$} & $\begin{array}{l}\text { aber ich frage mich OFT und das GRAde in meiner } \\
\text { tätigkeit an der beRUFSschule }\end{array}$ \\
\hline 02 & $(1.1)$ & \\
\hline 03 & {$[4,3]$} & WIE ich DEUTlich machen kann \\
\hline 4 & $(0.3)$ & \\
\hline 5 & {$[2,6]$} & $\begin{array}{l}\text { waRUM die bibel eben NICHT nur ein MÄRchenbuch } \\
\text { ist }\end{array}$ \\
\hline 6 & $(0.6)$ & \\
\hline & {$[4,6]$} & es steht so viel Gutes daRIN \\
\hline & $(0.7)$ & \\
\hline 9 & {$[4,8]$} & $\begin{array}{l}\text { was uns auch existentiell beTRIFFT aber wie KANN } \\
\text { der funke überspringen }\end{array}$ \\
\hline
\end{tabular}

Die Pfarrerin konstatiert, dass die Geltung der Bibel in den in ihr enthaltenen existentiell betreffenden Aussagen bestehe. Das Argumentationsziel wird durch eine hohe Betonungshäufigkeit in Verbindung mit sehr langsamer Artikulationsgeschwindigkeit in Zeile 05 emphatisch als relevant markiert (vgl. Schwitalla 2012:74, Uhmann 1992:330). Die Pfarrerin betont damit die Notwendigkeit und Möglichkeit, argumentativ hinsichtlich des Streitpunktes zu handeln. Es geht hier nicht mehr wie im vorherigen Beispiel um die Übernahme ihrer Haltung, sondern um die Argumentierbarkeit des richtigen Verständnisses der Bibel. Die Oratorin wechselt damit von der rhetorischen Ebene des Ethos auf die Ebene des Logos (vgl. Aristoteles 2002:1356a 19-21).

Im Anschluss verliest sie den Predigttext aus der Bibel, in dem der Autor die Geltung der Botschaft und das Entstehen der christlichen Gemeinde nicht von Fabeln, sondern von der existentiellen Erfahrung des Christusereignisses herleitet. Er 
verwendet dabei die Lichtmetaphorik, die bereits in vorhergehenden Teilen des Gottesdienstes angeklungen war. Für die Argumentation ist der Predigttext einerseits interessant, da die Geltung biblischer Texte ja selbst zur Debatte steht, andererseits, da die Geltung von Geschichten und Fabeln im Predigttext explizit verhandelt wird. Die Pfarrerin schließt daraus:

Beispiel (8): (Predigt Iserlohn Ausschnitt IV, 30:17-30:32)

\begin{tabular}{|c|c|c|}
\hline PFN & {$[5,6]$} & $\begin{array}{l}\text { da hat also jemand eine erFAHrung mit GOTT ge- } \\
\text { macht }\end{array}$ \\
\hline 02 & $(0.9)$ & \\
\hline 03 & {$[3,3]$} & und DAdurch sind ihm PLÖTZlich \\
\hline 4 & $(0.4)$ & \\
\hline 5 & {$[3,7]$} & TExte in der BIbel \\
\hline 6 & $(0.4)$ & \\
\hline 07 & {$[4,2]$} & ABsolut WICHtig geworden \\
\hline 8 & $(0.6)$ & \\
\hline 9 & {$[4,9]$} & $\begin{array}{l}\text { er hat gottes GEgenwart gespürt eine VoRahnung } \\
\text { von dem LICHT erhalten }\end{array}$ \\
\hline
\end{tabular}

Die Betonungshäufungen schließen an den Gestus der Lesung an. Schwitalla (2012:62) kennzeichnet einen solchen Übertrag prosodischer Muster in Anlehnung an Uhmann (1996:326) als Ansteckungsphänomen. Die Deutung des Predigttextes wird also der Sprechform nach an diesen angeglichen. Ein vergleichbares Phänomen war bereits bei dem oben angeführten Gebet aufgefallen. Dort hatte die Pfarrerin schon vor der Formel die rituelle Sprechhaltung eingenommen, hier folgt die rituell gefärbte Sprechform auf den verlesenen Text. Die Pfarrerin gibt ihre spontansprachliche, direkte Ansprechhaltung auf, gewinnt dabei aber selbst eine Nähe zum biblischen Text. Die dadurch gehobene Sprachform lässt deutlich die Verwendung der rhetorischen Ebene des Pathos (vgl. Aristoteles 2002:1356a 15-17) erkennen.

Wenig später schließt die Pfarrerin weiter, dass die existentiellen Erfahrungen, die sie als Inhalt der Bibel begreift, auch über die Entstehungszeit der Bibel hinaus Korrelate haben:

Beispiel (9): (Predigt Iserlohn Ausschnitt V, 32:33 - 32:43)

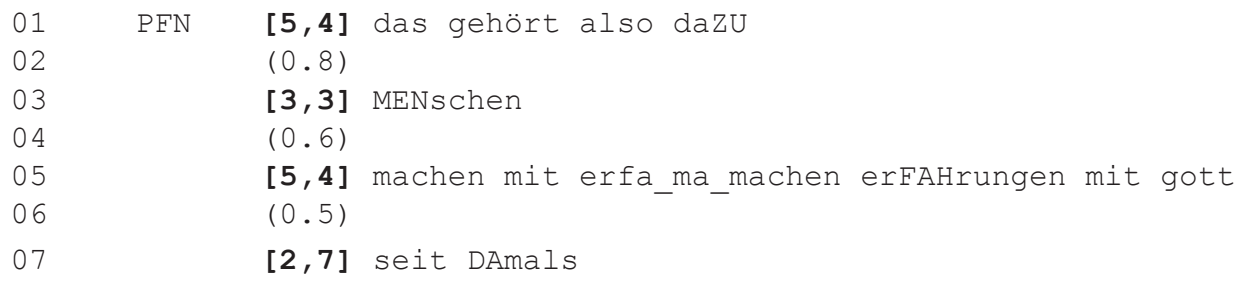


Durch die Lektüre der Bibel erschließe sich diese auch heute zugängliche Erfahrung der je einzelnen Leserin, dem je einzelnen Leser. Das zweitausend Jahre alte Zeugnis des Autors wird auf die heutige Zeit übertragen. Die Betonung der Geltung prinzipiell für alle Menschen wird besonders an der Emphase durch Reduktion der Artikulationsgeschwindigkeit (Z. 03) und die rahmende Pausierung (Z. 02, 04) deutlich.

Daraufhin überträgt die Pfarrerin die Aussage auf die Gemeinde und sich selbst.

Beispiel (10): (Predigt Iserlohn Ausschnitt VI, 33:34-33:46)

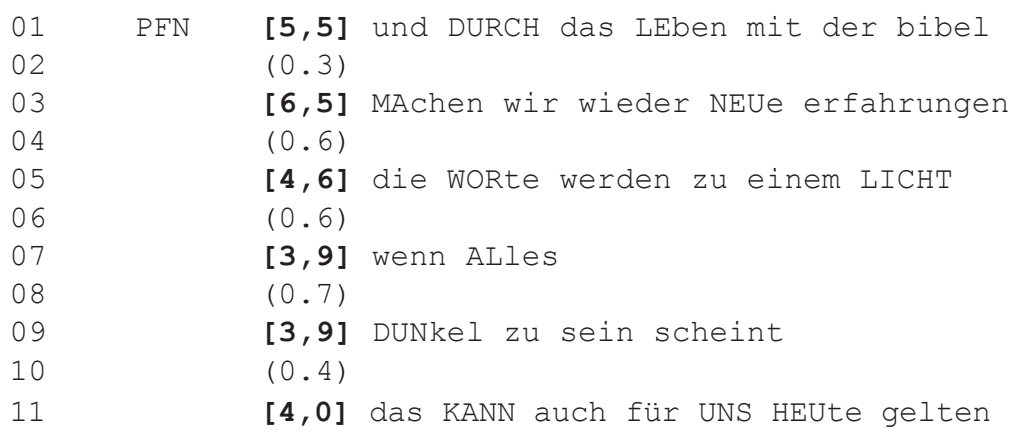

Am Eingang dieses Beispiels wechselt die Pfarrerin von der beschreibenden Ebene in der dritten Person wieder auf eine bekennende Ebene der ersten Person. Die beiden bekennenden Aussagen über die Gemeinschaft (Z. 03 und 11) rahmen dabei eine Aussage über die Geltung der Bibel, in der die Lichtmetaphorik wieder aufgenommen wird. Diese Aussage ist durch Pausengestaltung, reduzierte Artikulationsgeschwindigkeit und Betonungshäufungen als relevant markiert (vgl. Schwitalla 2012:74, Uhmann 1992:330). Die Pfarrerin bezieht dadurch ihre Hörerschaft in die Optionalität einer existentiellen Wirksamkeit der Bibel mit ein.

Die Pfarrerin untermauert schließlich die These der existentiellen Geltung der Bibel durch ein Beispiel.

Beispiel (11): (Predigt Iserlohn Ausschnitt VII, 33:54-34:06)

\begin{tabular}{|c|c|c|}
\hline $\begin{array}{l}01 \\
02\end{array}$ & $\begin{array}{l}{[4,5]} \\
(0.4)\end{array}$ & zum beispiel hat der sänger xavier naidoo \\
\hline 03 & {$[5,3]$} & SO zum GLAUben gefunden er hat das mal erZÄHLT \\
\hline 04 & $(0.4)$ & \\
\hline 05 & {$[6,1]$} & $\begin{array}{l}\text { in einer TALKshow er hat EINfach angefangen die } \\
\text { BIbel zu lesen }\end{array}$ \\
\hline & $(0.4)$ & \\
\hline . & {$[6,3]$} & und irgendwann \\
\hline 08 & $(0.6)$ & \\
\hline
\end{tabular}


09

10

11
$[6,4]$ hat er

$(0.3)$

$[3,7]$ gemerkt das betRIFFT mich

Die Pfarrerin stützt ihre bisher vorgetragene Argumentation, indem sie eine Person des öffentlichen Lebens als induktives Beispiel (vgl. Aristoteles 2002:1356b 1 -11) heranzieht. Auch wenn sie einen Einzelfall präsentiert, ist davon auszugehen, dass dieser die Argumentation stützt (zur Diskussion vgl. Schwarze 2010:66). Auch hier findet sich ein Topos aus der Person (Schwarze 2010:70f.). Um das Argument als schlüssig zu beurteilen, müssen die Hörer/-innen den Sänger dazu mit seiner öffentlichen Rolle als bekennendem Christen identifizieren. Die Pfarrerin spielt also auf das Weltwissen der Gemeinde an und schafft dadurch wiederum - wo das Beispiel verstanden wird - das Gefühl von Gruppenkohärenz. Die sehr hohe Artikulationsgeschwindigkeit, Anakoluth und Parenthese (Z. 03) deuten auf Spontansprache hin. Auffällig ist der plötzliche Abfall der Artikulationsgeschwindigkeit zur Pointe (Schwitalla 2012:74) in der letzten Phrase (Z. 11) hin.

Gegen Ende der Predigt stellt die Pfarrerin die Möglichkeit einer individuell positiven Wirkung der Auseinandersetzung mit der Bibel in Aussicht.

Beispiel (12): (Predigt Iserlohn Ausschnitt VIII, 37:09-37:22)

\begin{tabular}{|c|c|c|}
\hline $\mathrm{PFN}$ & $\begin{array}{l}{[4,8]} \\
(0,3)\end{array}$ & in den moMENten \\
\hline 03 & {$[5,1]$} & $\begin{array}{l}\text { wo wir auf gottes WORT ACHten und es in unser } \\
\text { innerstes FÄLLT }\end{array}$ \\
\hline & $(0.9)$ & \\
\hline & {$[3,7]$} & $\begin{array}{l}\text { DANN geht auch in UNsrem herzen der MORgenstern } \\
\text { auf }\end{array}$ \\
\hline & $(0.3)$ & \\
\hline 7 & {$[4,6]$} & $\begin{array}{l}\text { der TAG bricht AN wie es in dem } \\
\text { belvers eben HIEB }\end{array}$ \\
\hline
\end{tabular}

Die Pfarrerin knüpft die richtige Erkenntnis der Bibel an die Bedingung der Achtsamkeit (Z. 03). Wie schon im oben angeführten Schuldbekenntnis, steckt darin ein Appell an die Hörerinnen und Hörer. In Zeile 05 ist eine verringerte Artikulationsgeschwindigkeit von 3,7 Silben pro Sekunde (im Vergleich zu 5,1 Silben in Phrase 03 und 4,6 Silben in Phrase 07) zu verzeichnen. Verbunden mit der Betonungshäufung lässt dies wieder auf einen emphatischen Fokus auf die Relevanz dieser Phrase schließen (vgl. Schwitalla 2012:74, Uhmann 1992:330). Das Argumentationsziel, demzufolge die biblische Botschaft existentiell gültig ist, wird hier durch die Lichtmetapher unterstützt. Die Gültigkeit dieser Metapher sowie der gesamten These wird an die im vorhergehenden Beispiel erwiesene Geltung und Wirksamkeit der Bibel gebunden. 
Auf die Predigt folgen der Kanzelsegen, Gemeindegesang und Abkündigungen, ein Gebet und das Fürbittengebet und nochmals Gemeindegesang. Die anschließende Abendmahlsliturgie, innerhalb derer die Gemeinde zum Empfang von Brot und Wein eingeladen wird, endet mit einer Verbindung aus biblischem Text und individueller Gestaltung. Hier wird der Standpunkt, für den die Pfarrerin in der Predigt argumentiert hat, noch einmal gestützt.

Beispiel (13): (Sendung nach Abendmahl Iserlohn, 01:02:18-01:02:27)

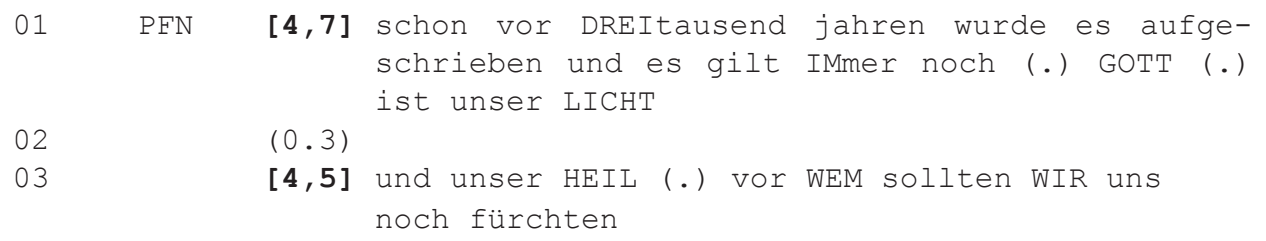

Die Funktion dieser sog. Sendung ist es, die Gemeindeglieder mit einem Segen wieder auf ihre Plätze zu entlassen. Die Pfarrerin beansprucht im Sinne des gesamten Argumentationszusammenhangs, dass der von ihr zitierte Bibeltext (angelehnt an einen biblischen Psalm) unmittelbar und auch für die anwesende Gemeinde gilt (Z. 01). Der Zusammenhang zur vorherigen Argumentation wird durch die Aufnahme der Lichtmetaphorik hergestellt. Damit wird zugleich ein Bogen hin zur Begrüßungsmoderation geschlagen. Der Bibeltext wird hier ferner nicht als Lesung, sondern als freie Rezitation vorgetragen, was an der relativ hohen Artikulationsgeschwindigkeit und der Einbindung in eine kommentierende Vorrede erkennbar wird. Diese zuletzt zitierte Passage ist aufgrund der besonderen räumlichen Nähe der Pfarrerin zu den Gemeindegliedern und als letzter frei formulierter Text des Gottesdienstes von besonderer Bedeutung. Sie bildet den Abschluss des Argumentationszusammenhangs, der den gesamten Gottesdienst durchzieht und erfolgt nicht mehr in der Form eines Anspruchs oder einer Abwägung, sondern als Zuspruch.

Den Abschluss des Gottesdienstes bilden ein Lied, die Sendungsworte und der Segen in ihrer agendarisch vorgegebenen Gestalt, das Amen der Gemeinde und Instrumentalmusik.

Die gottesdienstliche Makroargumentation zur Geltung der Bibel, die hier zu beobachten ist, zieht sich durch verschiedene gottesdienstliche Strukturelemente und unterschiedliche Texttypen. Dabei nutzen die Beteiligten das Potential jedes einzelnen Strukturelements und jeder einzelnen Textsorte in ihrem Sinne: In der Begrüßung wird der Streitpunkt bereits mit einer Tendenz gesetzt. Das Schuldbekenntnis wird genutzt, um für die Hörerinnen und Hörer einen gemeinsamen Standpunkt zu etablieren. Damit wird die weitere Argumentation vorbereitet. Im Gebet variiert die Pfarrerin den Standpunkt dann insofern weiter, als über göttliche Hilfe hinaus die Möglichkeit eines eigenen Mitwirkens am Wechsel des Standpunkts eröffnet wird. Die erkenntnistheoretische Fragestellung nach dem 
Wissen von der Geltung biblischer Texte wird hierbei erweitert zu einer ethischen Fragestellung nach dem richtigen Umgang mit der Bibel. In der Predigt führt die Pfarrerin dann durch unter Bezug auf Verstand (Logos) und Affekt (Ethos und Pathos), sowie unter Verwendung argumentativer Topoi (aus der Autorität, aus der Person, Beispiel) die Gemeinde auf das Argumentationsziel hin. In den Sendungsworten nach dem Abendmahl demonstriert sie schließlich die Geltung der diskutierten These. Auf der sprechakttheoretischen Ebene ist schließlich der Wechsel zwischen konstativen und performativen Akten auffällig. In Schuldbekenntnis und Segen werden Haltungen und Einstellungen nicht nur argumentativ diskutiert, sondern sie werden vielmehr performativ hervorgebracht.

\section{Resümee}

Am Beginn dieses Beitrags stand eine Alternative: Gottesdienst, der verkürzt als Predigt verstanden wird, ist auf Persuasion ausgerichtet. Gottesdienst, der verkürzt mit der Liturgie identifiziert wird, ist es nicht. Diese Alternative wurde bereits im ersten Hauptteil aufgebrochen. Es wurde gezeigt, dass und wie in verschiedenen Zeiten und Kontexten unterschiedliche hermeneutische Zugriffe auf das Phänomen Gottesdienst versucht wurden. Für eine rhetorische Betrachtung der Gesamtgestalt des Gottesdienstes wurde aus Theologie, Rhetorik und Ritualtheorie ein Persuasionsbegriff entwickelt, der dem Gegenstand angemessen ist. Dabei wurde dargestellt, dass der evangelische Gottesdienst aus einer Vielzahl von Elementen besteht, die für sich allein und im Zusammenspiel mehr oder weniger Gelegenheit bieten, persuasiv zu argumentieren. Wo das Ritual in den Hintergrund tritt, ist die Kommunikation für die Wahl rhetorischer Zwecke und Mittel offen. Das gilt nicht nur für die Predigt. Dies wurde an zwei Gottesdiensten, wie sie jeden Sonntag tausendfach in Kirchen stattfinden, illustriert. Der Fokus lag dabei einmal auf der Mikroebene der Argumentation und wurde im zweiten Beispiel auf die Makroebene ausgeweitet. Es wurde dargestellt, wie spontansprachliche Argumentation mit alltagsweltlicher Zielsetzung ein Teil eines evangelischen Gottesdienstes sein kann und wie sie dazu verhelfen kann, Koordinierungsprobleme in einer Gemeinschaft zu bearbeiten. Der Zweck der Argumentation lag außerhalb des Gottesdienstes, aber in der Beförderung des gemeindlichen Zusammenlebens. Im zweiten Fall hatte die nachgezeichnete Argumentation ein explizit religiöses Ziel, das mit explizit theologischen Argumenten verfolgt wurde. Es zeigte sich, dass ein ganzer Gottesdienst von einer übergreifenden Argumentation durchzogen sein kann und dass das Beweisziel von verschiedenen Personen zusammen verfolgt werden kann. Besonders interessant war dabei die Grenzlinie zwischen ritueller und alltagsweltlicher Sprach- und Sprechform. Das Nebeneinander von ritueller und rhetorischer Haltung und Sprache kann zu besonderen Effekten führen. Das wurde deutlich, wo rituelle sprecherische Muster in einen nicht-rituellen Kontext 
übernommen wurden. Die Verteilung argumentativer Sprechhandlungen über einen gesamten Gottesdienst und auf mehrere Akteurinnen bzw. Akteure ermöglicht es, wie sich zeigte, einen Streitpunkt zu entwickeln und ihn aus unterschiedlichen Blickwinkeln argumentativ zu beleuchten und die Hörerschaft in verschiedenen Modi anzusprechen. Aus kommunikationsethischer Perspektive können hierbei aber auch Grenzüberschreitungen beobachtet werden, wenn die rituelle Gestalt so mit der argumentativ-rhetorischen verknüpft wird, dass freie Argumentation aufseiten des Orators bzw. der Oratorin auf Zwangskommunikation rituellen Einverständnisses bei den Hörerinnen und Hörern trifft. Andere Argumentationsformen stellten etwa den Bezug zu Gefühl und Verstand der Gottesdienstbesucherinnen und Gottesdienstbesucher her.

Die Analyse der Sprache und der rhetorischen Strategie in evangelischen Gottesdiensten wurde gewinnbringend mit der Analyse sprecherischer Faktoren, besonders des Einsatzes von Betonungen, Pausierung und Artikulationsgeschwindigkeit, verbunden. Dies verhalf dazu, Vermutungen zu Intention und Wirkung der Kommunikation aufzustellen. Bei alledem ist stets zu beachten, dass die hier durchgeführte Analyse explorativen Charakter hat. Sie zeigt, dass der Einbezug prosodischer Merkmale in die Analyse gottesdienstlicher Argumentation zu vertieften Erkenntnissen führen kann. Künftige Untersuchungen hätten nun die Aufgabe, die hier aufgestellten Vermutungen an weiteren Beispielen zu erhärten. Ferner wäre es denkbar, die Daten in Zukunft um weitere Merkmale wie Intonationsverläufe, und artikulatorische Merkmale zu vermehren.

Der evangelische Gottesdienst ist ein aus empirisch-linguistischer, sprechwissenschaftlicher und rhetorischer Sicht noch weitgehend unbestelltes wissenschaftliches Feld. Mehr noch als zur Analyse von Predigten bedarf es bei der Betrachtung des gottesdienstlichen Gesamtzusammenhangs eines interdisziplinären Zugangs. Eine besondere Herausforderung besteht darin, eine angemessene Beschreibungssprache zu entwickeln. Diese Beschreibungssprache muss anschlussfähig sein an die Weltdeutung, die sich im gottesdienstlichen Handeln vollzieht. Sie muss die Praxis des Gottesdienstes als Arbeit an immanentem und transzendentem Sinn wahrnehmen. Dazu ist sie auf kulturwissenschaftliche und theologische Hermeneutiken angewiesen.

Derart fundiert können weitere Studien einen wichtigen Beitrag zur Orientierung über das innere Aptum des Gottesdienstes beitragen. Damit beleuchten sie eine Wirklichkeit, die Agendenwerke mit ihrem Fokus auf das äußere Aptum beiseite lassen

\section{Literatur}

ARISTOTELES, 2002, Rhetorik, in: Rapp Chr. (Hrsg.), Aristoteles, Werke in deutscher Übersetzung, 4/II, Berlin. 
ATAYAN Vahram, 2006, Makrostrukturen der Argumentation im Deutschen, Französischen und Italienischen, Frankfurt a. M./New York.

BIERITZ Karl-Heinrich, 1998, Spielraum Gottesdienst, Von der „Inszenierung des Evangeliums“ auf der liturgischen Bühne, in: Schilson A./Hake J. (Hrsg.), Drama „Gottesdienst“. Zwischen Inszenierung und Kult, Stuttgart, S. 69-101.

BIERITZ Karl-Heinrich, 2004, Liturgik, Berlin/New York.

Evangelische Kirche in Deutschland (Hrsg.), 2014, gezählt, Zahlen und Fakten zum kirchlichen Leben. Online verfügbar unter https://www.ekd.de/download/zahlen_und_fakten_2014.pdf, zuletzt geprüft am 10.04.2016.

FISCHER-LiCHTE Erika, 2006, Kirchenräume als performative Räume, in: Artheon - Mitteilungen der Gesellschaft für Gegenwartskunst und Kirche (24), S. 19-25, www.artheon.de/fileadmin/ bilder/download/Mitteilungen/Artheon24.pdf, zuletzt geprüft am 10.03.2016.

FRIEDRICH Marcus A., 2001, Liturgische Körper, Der Beitrag von Schauspieltheorien und -techniken auf die Pastoralästhetik, Stuttgart.

GEISSLER Ewald, 1925, Vom Vortrag des Heiligen, in: Monatsschrift für Gottesdienst und kirchliche Kunst, S. 189-197.

GEISSNER Hellmut, 1977, Die Predigt und die rhetorische Kommunikation im Gottesdienst, in: Praktische Theologie 12(1), S. 48-57.

GRÄB Wilhelm, 1997, Der inszenierte Text. Erwägungen zum Aufbau ästhetischer und religiöser Erfahrung in Gottesdienst und Predigt, in: International Journal of Practical Theology 1(2), S. 209-226.

GRÄB Wilhelm, 2013, Predigtlehre, Über religiöse Rede, Göttingen.

GRAFF Paul, 1994, Geschichte der Auflösung der alten gottesdienstlichen Formen in der evangelischen Kirche Deutschlands, Band 1: Bis zum Eintritt der Aufklärung und des Rationalismus, Unveränderter Nachdruck der zweiten Ausgabe 1937, Waltrop.

GRETHLEIN Christian, 2012, Praktische Theologie, Berlin/Boston.

GRÖZINGER Albrecht, 2007, Art. Rhetorik, Kunst der Rede/Religiöse Sprache/Sprechakttheorie/ Homiletik und Rhetorik, in: Gräb W./Weyel B. (Hrsg.), Handbuch Praktische Theologie, Gütersloh, S. 821-832.

HAUSENDORF Heiko / SCHMITT Reinhold, 2014, Vier Stühle vor dem Altar, Eine interaktionslinguistische Fallstudie zur Raumnutzung in einem „Alpha-Gottesdienst“, Zürich (Arbeitspapiere des UFSP Sprache und Raum (SpuR), 3). Online verfügbar unter www.spur.uzh.ch/research/ SpuR_Arbeitspapier_Nr03_140702.pdf.

HUMPHREY Caroline / LAIDLAW James, 1994, The Archetypal Actions of Ritual, a Theory of Ritual Illustrated by the Jain Rite of Worship, Oxford/New York.

HuMPHREY Caroline / LAIDLAW James, 2008, Die rituelle Einstellung, in: Belliger A./Krieger D.J. (Hrsg.), Ritualtheorien, Ein einführendes Handbuch, 4. Auflage, Wiesbaden, S. 135-155.

KIRCHENLEITUNG der Vereinigten Evangelisch-Lutherischen Kirche Deutschlands / Kirchenkanzlei der Evangelischen Kirche der Union (im Auftrag des Rates) (Hrsg.), 2000, Evangelisches Gottesdienstbuch, Agende für die Evangelische Kirche der Union und für die Vereinigte Evangelisch-Lutherische Kirche Deutschlands, 3. Auflage, Berlin.

KuIE Thomas, 2009, Vom Ritual zum Ritus, Ritologische Schneisen im liturgischen Dickicht, in: Berliner Theologische Zeitschrift 26(1), S. 96-107.

KLIE Thomas, 2010, Fremde Heimat Liturgie, Ästhetik gottesdienstlicher Stücke, Stuttgart.

KLoss Ulla, 2002, Moderation des Gottesdienstes, Der Beitrag moderierender Äußerungen für die Konstitution gottesdienstlicher Gemeinschaft und das Gelingen des Gottesdienstes, DGSS-Abschlussarbeit. Philipps-Universität, Marburg.

KNAPE Joachim, 2003, Art. Persuasion, in: Ueding G. (Hrsg.), Historisches Wörterbuch der Rhetorik, Band 6., Tübingen, Sp. 874-907. 
KNAPE Joachim, 2010, Rhetorik und Predigt, Wie viel Rhetorik braucht die Predigt?, in: MeyerBlanck M./Spielberg B./Seip J. (Hrsg.), Homiletische Präsenz. Predigt und Rhetorik, München, S. 29-51.

KNAPE Joachim, 2013, Predigt als Kunst, Rhetorik oder Kreativakt, Moderne Predigtlehre und Christian Palmers Homiletik, in: Drehsen V./Schweitzer F./Weyel B. (Hrsg.), Christian Palmer und die Praktische Theologie, Jena, S. 11-51.

Kowal Sabine, 1991, Über die zeitliche Organisation des Sprechens in der Öffentlichkeit, Pausen, Sprechtempo und Verzögerungen in Interviews und Reden von Politikern, Bern/Stuttgart/ Toronto.

MARTIN Gerhard Marcel, 1984, Predigt als „offenes Kunstwerk“?, Zum Dialog zwischen Homiletik und Rezeptionsästhetik, in: Evangelische Theologie 44, S. 46-58.

MEINHOLD Gottfried, 1995, Zeitparameter gesprochener Sprache, Forschungsbericht, Unveröffentlichtes Manuskript. Universität Jena, Lehrstuhl für Phonetik und Sprechwissenschaft, Jena.

MEYER-BlancK Michael, 2012, Gottesdienstlehre, Tübingen.

O'Connell Daniel C. / Kowal Sabine, 2008, Communicating with One Another, Toward a Psychology of Spontaneous Spoken Discourse, New York.

Отто Gert, 1976, Die Predigt als Rede, Über die Wechselwirkungen von Homiletik und Rhetorik, Stuttgart.

Отто Gert, 1986, Zur Kritik am rhetorischen Predigtverständnis, in: Dyck J./Jens W./Ueding G. (Hrsg.), Rhetorik, ein internationales Jahrbuch, Band 5, Rhetorik und Theologie. Tübingen, S. $1-12$.

PALMER Christian, 1842, Evangelische Homiletik, Stuttgart.

PALmer Christian, 1845, Evangelische Homiletik, 2. Auflage, Stuttgart.

PFITZINGER Hartmut R., 2001, Phonetische Analyse der Sprechgeschwindigkeit, in: Forschungsberichte des Instituts für Phonetik und Sprachliche Kommunikation der Universität München (38), S. 117-264, Dissertation. Online verfügbar unter http://phonetik.uni-muenchen.de/forschung/FIPKM/vol38/f38_hp_1.pdf, zuletzt geprüft am 08.03.2016.

Rотн Ursula, 2006, Die Theatralität des Gottesdienstes, Gütersloh.

SCHULZ Lars-Robin, 2014, Merkmale gesprochener Sprache im evangelischen Gottesdienst, unveröffentlichte Masterthesis. Martin-Luther-Universität, Halle-Wittenberg.

SCHWARZE Cordula, 2010, Formen und Funktionen von Topoi im Gespräch, Eine empirische Untersuchung am Schnittpunkt von Argumentationsforschung, Gesprächsanalyse und Sprechwissenschaft, Frankfurt a. M. u.a.

SCHWEINSBERG Fritz, 1946, Stimmliche Ausdrucksgestaltung im Dienste der Kirche, Ein Werkbuch für die Wiederaufbauarbeit, Heidelberg.

SCHWIER Helmut / GALl Sieghard, 2008, Predigt hören, Befunde und Ergebnisse der Heidelberger Umfrage zur Predigtrezeption, Münster.

SCHWitalla Johannes, 2012, Gesprochenes Deutsch, Eine Einführung, 4., neu bearbeitete und erweiterte Auflage, Berlin.

Selting Margret / Auer Peter / BARTh-Weingarten Dagmar / Bergmann Jörg / Bergmann Pia / BIRKNER Karin / COUPER-KUHLEN Elizabeth / DEPPERMANN Arnulf/ GILles Peter / GÜNTHNER Susanne / HARTUng Martin / Kern Friederike / Mertzlufft Christine / MeYer Christian / MoreK Miriam / OBerzaucher Frank / Peters Jörg / QuAsthoff Uta / SchÜtTE Wilfried / StUKEnBrock Anja / Uhmann Susanne, 2009, Gesprächsanalytisches Transkriptionssystem 2 (GAT 2), in: Gesprächsforschung - Online-Zeitschrift zur verbalen Interaktion, Ausgabe 10, S. $353-402$ (www.gespraechsforschung-ozs.de). 
THIELE Michael, 2004, Geistliche Beredsamkeit, Reflexionen zur Predigtkunst, Stuttgart.

THURNEYSEN Eduard, 1971, Die Aufgabe der Predigt, in: Hummel G. (Hrsg.), Aufgabe der Predigt, Darmstadt, S. 105-118.

Uhmann Susanne, 1992, Contextualizing Relevance, On Some Forms and Functions of Speech Rate Changes in Everyday Conversation, in: Auer P./Di Luzio, A. (Hrsg.), The Contextualization of Language, Amsterdam/Philadelphia, S. 297-336.

Uhmann Susanne, 1996, On Rhythm in Everyday German Conversation, Beat Clashes in Assessment Utterances, in: Couper-Kuhlen, E./Selting, M. (Hrsg.), Prosody in Conversation. Interactional Studies, Cambridge/New York, S. 303-365.

\section{Internetquellen (IQ)}

1. Gottesdienst auf Kassette. Online verfügbar unter www.ekir.de/euskirchen/altenarbeit/gottesdienstekassette.htm, zuletzt geprüft am 16.10.2015.

2. f4: https://www.audiotranskription.de/f4.htm, zuletzt geprüft am 05.03.2016.

3. Praat: doing phonetics by computer. Online verfügbar unter www.fon.hum.uva.nl/praat/, zuletzt geprüft am 05.03.2016.

4. find_pauses. Online verfügbar unter praatpfanne.lingphon.net/downloads/1_find_pauses.txt, zuletzt geprüft am 05.03.2016. 